Supplementary materials for:

\title{
Does the "Superatom" Exist in Halogenated Aluminum Clusters?
}

\author{
Young-Kyu Han* and Jaehoon Jung
}

Computational Chemistry Laboratory, Corporate R\&D, LG Chem, Ltd. Research Park 104-1, Moonji-dong, Yuseong-gu, Daejeon 305-380, South Korea 


\section{Contents}

\section{Computation Details}

Figure S1. Ionization potentials and electron affinities for $\mathrm{Al}_{n}(n=11-15)$.

Figure S2. Optimized structures and relative energies for $\mathrm{Al}_{n}^{q}(n=11-15, q=-1,0,+1$, and +2$)$.

Figure S3. Optimized structures and relative energies for $\mathrm{Al}_{n} \mathrm{X}(n=11-15, \mathrm{X}=\mathrm{F}, \mathrm{Cl}, \mathrm{Br}$, and $\mathrm{I})$.

Figure S4. Optimized structures and relative energies for $\mathrm{Al}_{n} \mathrm{X}_{2}(n=11-15, \mathrm{X}=\mathrm{F}, \mathrm{Cl}, \mathrm{Br}$, and I).

Table S1. (a) Charge $(Q)$ and electronegativity $(\eta)$ of $\mathrm{MX}\left(\mathrm{M}=\mathrm{Al}, \mathrm{Al}_{11}-\mathrm{Al}_{15}\right.$, Halogen atoms, $\left.\mathrm{X}=\mathrm{F}, \mathrm{Cl}, \mathrm{Br}, \mathrm{I}\right)$; (b) Charge $(Q)$ and electronegativity $(\eta)$ of $\mathrm{MX}_{2}\left(\mathrm{M}=\mathrm{Al}, \mathrm{Al}_{11}-\mathrm{Al}_{15}, \mathrm{Si}\right.$, Alkaline earth atoms, $\left.\mathrm{X}=\mathrm{F}, \mathrm{Cl}, \mathrm{Br}, \mathrm{I}\right) ;(\mathrm{c})$ Optimized structures of MX (M=Al, Halogen atoms, $\mathrm{X}=\mathrm{F}, \mathrm{Cl}, \mathrm{Br}, \mathrm{I})$; (d) Optimized structures of $\mathrm{MX}_{2}(\mathrm{M}=\mathrm{Al}, \mathrm{Si}$, Alkaline earth atoms, $\mathrm{X}=\mathrm{F}, \mathrm{Cl}, \mathrm{Br}, \mathrm{I})$.

Figure S5. Optimized structures and relative energies for $\mathrm{Al}_{n} \mathrm{I}_{2}^{-}(n=11-15)$.

Figure S6. Optimized structures and relative energies for $\mathrm{Al}_{n} \mathrm{I}_{3}{ }^{-}(n=11-15)$.

Table S2. Charge distribution $Q\left(\mathrm{Al}_{n}\right)$ for $\mathrm{Al}_{n} \mathrm{I}_{2}{ }^{-}$and $\mathrm{Al}_{n} \mathrm{I}_{3}^{-}(n=11-15)$.

Figure S7. $\mathrm{HOMO}-\mathrm{LUMO}$ gaps for $\mathrm{Al}_{n} \mathrm{I}_{2}{ }^{-}$and $\mathrm{Al}_{n} \mathrm{I}_{3}{ }^{-}(n=11-15)$.

Figure S8. Molecular orbital diagram for $\mathrm{Al}_{13}{ }^{-}$and $\mathrm{Al}_{13} \mathrm{~K}^{-}$.

Figure S9. Molecular orbital diagram for $\mathrm{Al}_{15}{ }^{-}$and $\mathrm{Al}_{15} \mathrm{I}_{2}{ }^{-}$.

Table S3. Absolute energies (in Hartrees) and Cartesian coordinates for $\mathrm{Al}_{n}{ }^{q}(n=11-15, q=-1,0,+1$, and +2$)$, $\mathrm{Al}_{n} \mathrm{X}\left(n=11-15, \mathrm{X}=\mathrm{F}, \mathrm{Cl}, \mathrm{Br}\right.$, and I), $\mathrm{Al}_{n} \mathrm{X}_{2}(n=11-15, \mathrm{X}=\mathrm{F}, \mathrm{Cl}, \mathrm{Br}$, and $\mathrm{I}), \mathrm{Al}_{n} \mathrm{I}^{-}, \mathrm{Al}_{n} \mathrm{I}_{2}^{-}$, and $\mathrm{Al}_{n} \mathrm{I}_{3}{ }^{-}(n=$ $11-15)$. 


\section{Complete reference 9(d)}

Purath, A.; Dohmeier, C.; Ecker, A.; Köppe. R.; Krautscheid, H.; Schnöckel, H.; Ahlrichs, R.; Stoermer, C.; Friedrich, J.; Jutzi, P. J. Am. Chem. Soc. 2000, 122, 6955-6959.

\section{Computation Details}

For each specific cluster size, an exhaustive search for minimum energy structures was performed. We selected 30-160 initial geometry configurations without any symmetry constraint for geometry optimization. Kohn-Sham density functional theory (DFT) calculations were performed with the gradient-corrected exchange-correlation functional proposed by Perdew, Burke, and Ernzerhof (PBE). ${ }^{[1]}$ The correlationconsistent triple- $\zeta$ (cc-pVTZ) basis set was used. Scalar relativistic effects were treated with the energyconsistent pseudopotentials for the heavy $\mathrm{Br}$ and I atoms. ${ }^{[2]}$ Natural population analysis $(\mathrm{NPA})^{[3]}$ calculations were performed to estimate quantitatively the charge distribution. All the calculations were carried out with the Gaussion03 program. ${ }^{[4]}$

[1] Perdew, J. P.; Burke, K.; Ernzerhof, M. Phys. Rev. Lett. 1996, 77, 3865-3868.

[2] Peterson, K. A.; Figgen, D.; Goll, E.; Stoll, H.; Dolg, M. J. Chem. Phys. 2003, 119, 11113-11123.

[3] Reed, A. E.; Weinstock, R. B.; Weinhold, F. J. Chem. Phys. 1985, 83, 735-746.

[4] Gaussian03, Revision C.02, Frisch, M. J.; Trucks, G. W.; Schlegel, H. B.; Scuseria, G. E.; Robb, M. A.; Cheeseman, J. R.; Montgomery, J., J. A.; Vreven, T.; Kudin, K. N.; Burant, J. C.; Millam, J. M.; Iyengar, S. S.; Tomasi, J.; Barone, V.; Mennucci, B.; Cossi, M.; Scalmani, G.; Rega, N.; Petersson, G. A.; Nakatsuji, H.; Hada, M.; Ehara, M.; Toyota, K.; Fukuda, R.; Hasegawa, J.; Ishida, M.; Nakajima, T.; Honda, Y.; Kitao, O.; Nakai, H.; Klene, M.; Li, X.; Knox, J. E.; Hratchian, H. P.; Cross, J. B.; Bakken, V.; Adamo, C.; Jaramillo, J.; Gomperts, R.; Stratmann, R. E.; Yazyev, O.; Austin, A. J.; Cammi, R.; Pomelli, C.; Ochterski, J. W.; Ayala, P. Y.; Morokuma, K.; Voth, G. A.; Salvador, P.; Dannenberg, J. J.; Zakrzewski, V. G.; Dapprich, S.; Daniels, A. D.; Strain, M. C.; Farkas, O.; Malick, D. K.; Rabuck, A. D.; Raghavachari, K.; Foresman, J. B.; Ortiz, J. V.; Cui, Q.; Baboul, A. G.; Clifford, S.; Cioslowski, J.; Stefanov, B. B.; Liu, G.; Liashenko, A.; Piskorz, P.; Komaromi, I.; Martin, R. L.; Fox, D. J.; Keith, T.; Al-Laham, M. A.; Peng, C. Y.; Nanayakkara, A.; Challacombe, M.; Gill, P. M. W.; Johnson, B.; Chen, W.; Wong, M. W.; Gonzalez, C.; Pople, J. A. Gaussian, Inc., Wallingford CT, 2004. 
Figure S1. Ionization potentials and electron affinities for $\mathrm{Al}_{n}(n=11-15)$. A: Adiabatic, V: Vertical.

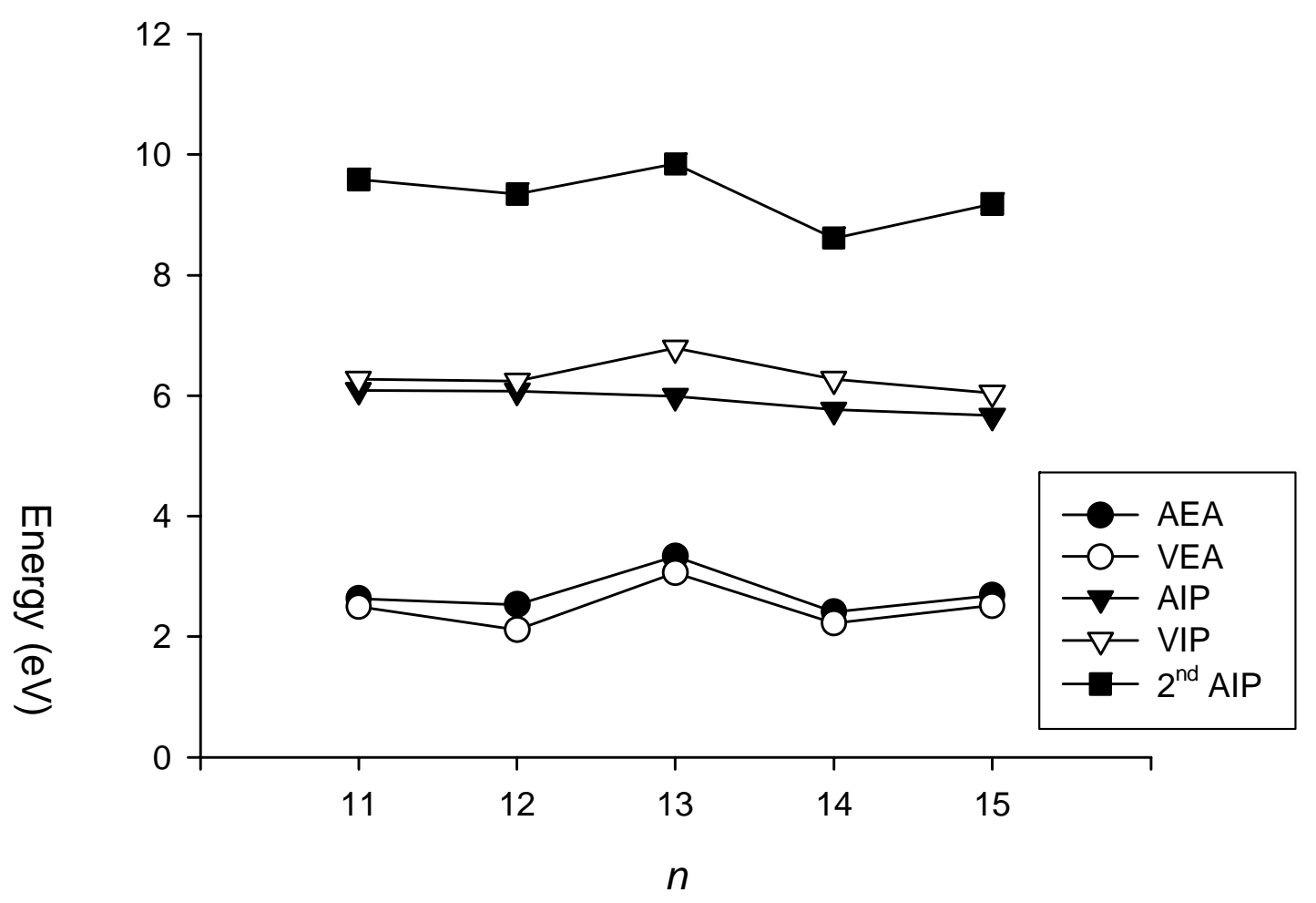

$\mathrm{Al}_{13}$ has 39 valence electrons, and thus $\mathrm{Al}_{13}$ should have a very high $\mathrm{EA}$. Indeed, this has been demonstrated experimentally; $\mathrm{Al}_{13}$ has an $\mathrm{EA}$ of $3.6 \mathrm{eV},{ }^{[5]}$ which is similar to that of $\mathrm{Cl}(3.61 \mathrm{eV})$, the highest $\mathrm{EA}$ among the elements in the Periodic Table. Furthermore, $\mathrm{Al}_{13}{ }^{-}$has a very high-symmetry icosahedral structure, rendering it both electronically and geometrically stable. We note that the adiabatic IP values decrease monotonically with an increase in $n$ for $\mathrm{Al}_{n}$ clusters, while $\mathrm{Al}_{13}$ has the greatest vertical IP. For $\mathrm{Al}_{14}$ clusters, the second IP value is smaller than those of other clusters, and $\mathrm{Al}_{14}$ adopts a spherical shape in $\mathrm{Al}_{14}{ }^{2+}$. Both the $\mathrm{Al}_{13}{ }^{-}$and $\mathrm{Al}_{14}{ }^{2+}$ clusters are stable and spherical, which may be explained in terms of the spherical jellium model. One can conclude that $\mathrm{Al}_{13}$ and $\mathrm{Al}_{14}$ have superhalogen and superalkaline earth characteristics, respectively.

[5] Li, X.; Wu, H.; Wang, X.-B.; Wang, L.-S. Phys. Rev. Lett. 1998, 81, 1909-1912. 
Figure S2. Optimized structures and relative energies (in $\mathrm{eV})$ for $\mathrm{Al}_{11}{ }^{q}(q=-1,0,+1$, and +2$)$.

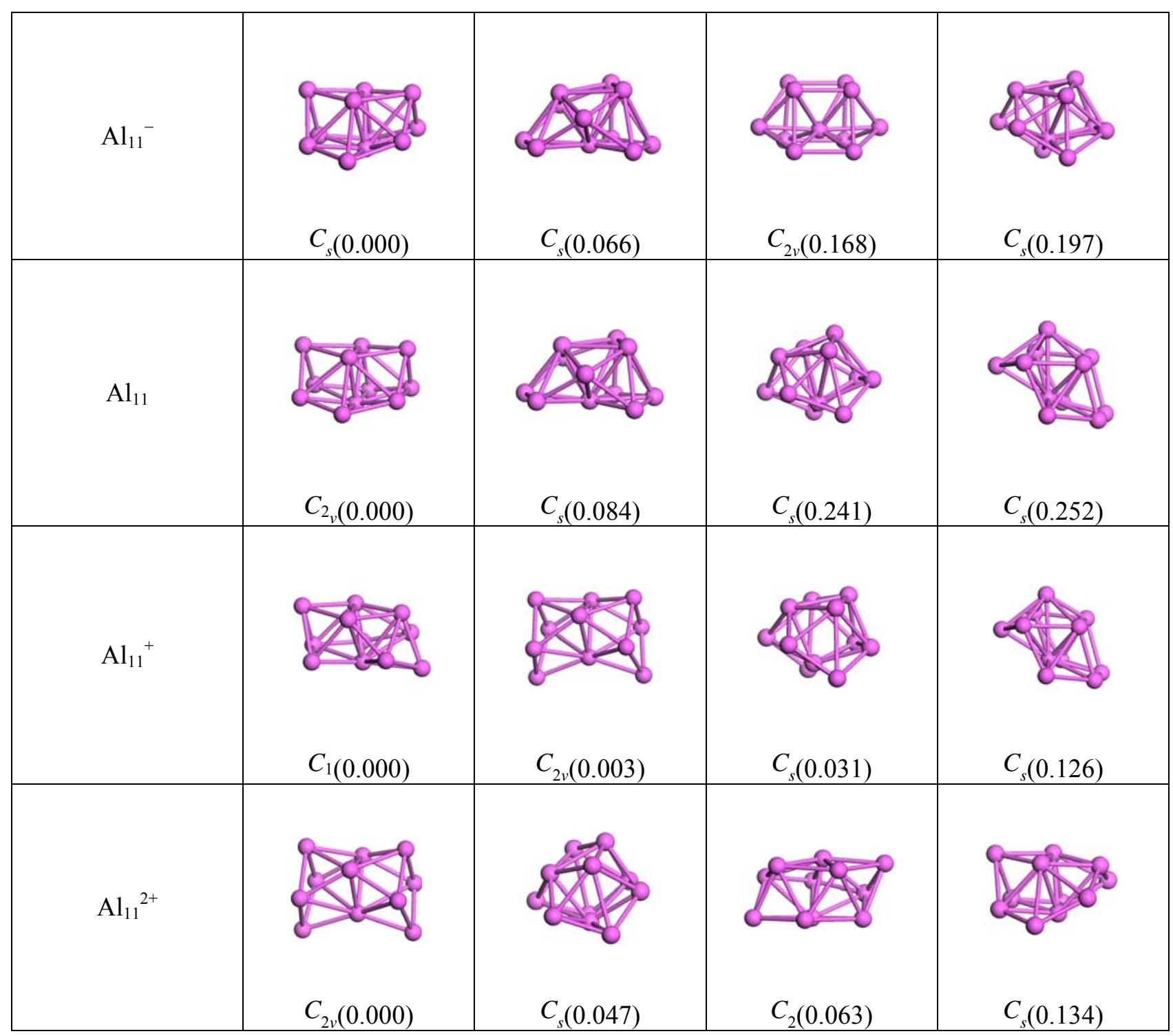


- Optimized structures and relative energies (in $\mathrm{eV})$ for $\mathrm{Al}_{12}{ }^{q}(q=-1,0,+1$, and +2$)$.

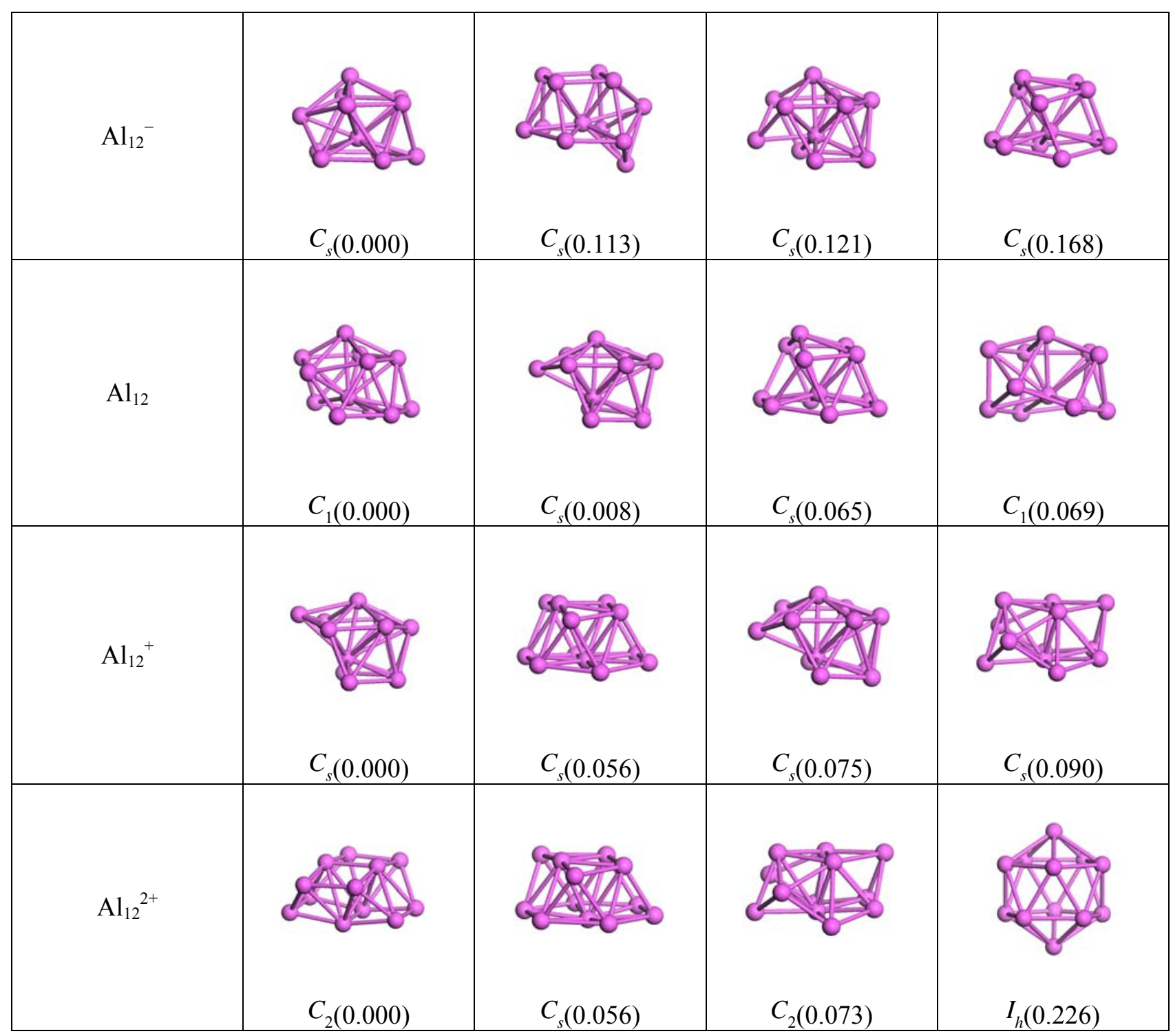


- Optimized structures and relative energies (in $\mathrm{eV})$ for $\mathrm{Al}_{13}{ }^{q}(q=-1,0,+1$, and +2$)$.

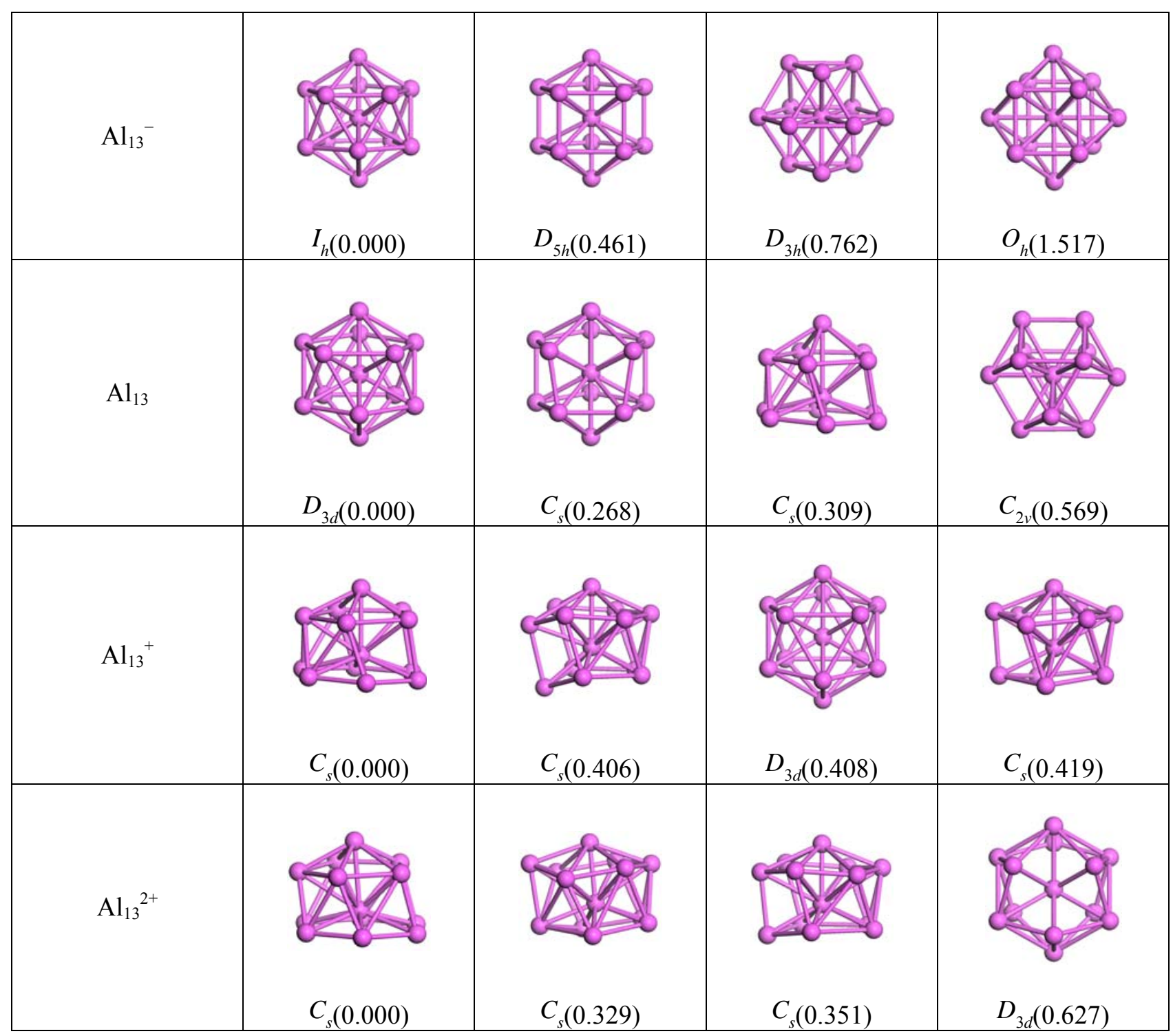


- Optimized structures and relative energies (in $\mathrm{eV})$ for $\mathrm{Al}_{14}{ }^{q}(q=-1,0,+1$, and +2$)$.

\begin{tabular}{|c|c|c|c|c|}
\hline $\mathrm{Al}_{14}^{-}$ & $C_{s}(0.000)$ & $\frac{C_{2 v}(0.228)}{21}$ & $C_{s}(0.460)$ & $C_{3 v}(0.665)$ \\
\hline $\mathrm{Al}_{14}$ & $C_{3 v}(0.000)$ & $C_{2_{v}}(0.054)$ & $C_{2_{v}}(0.128)$ & $C_{s}(0.430)$ \\
\hline $\mathrm{Al}_{14}^{+}$ & $C_{2_{v}}(0.000)$ & $C_{3 v}(0.007)$ & $C_{2_{\nu}}(0.074)$ & $C_{3 v}(0.120)$ \\
\hline $\mathrm{Al}_{14}{ }^{2+}$ & $C_{3 v}(0.000)$ & $C_{s}(0.038)$ & $C_{2_{v}}(0.039)$ & $C_{2_{v}}(0.318)$ \\
\hline
\end{tabular}


- Optimized structures and relative energies (in $\mathrm{eV})$ for $\mathrm{Al}_{15}{ }^{q}(q=-1,0,+1$, and +2$)$.

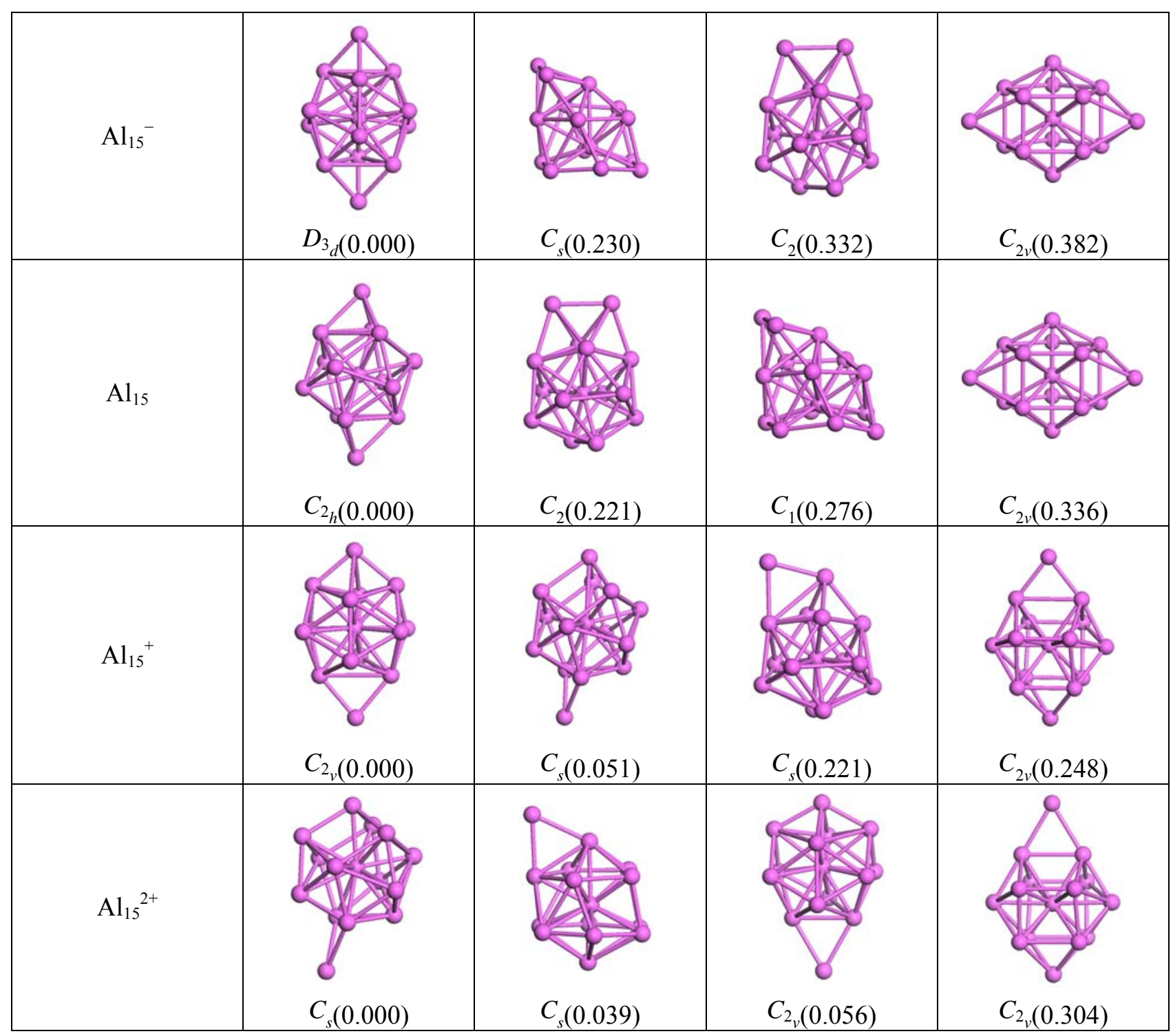


Figure S3. Optimized structures and relative energies (in $\mathrm{eV})$ for $\mathrm{Al}_{11} \mathrm{X}(\mathrm{X}=\mathrm{F}, \mathrm{Cl}, \mathrm{Br}$, and $\mathrm{I})$.

\begin{tabular}{|c|c|c|c|c|}
\hline & & & \\
& $C_{s}$ & $C_{1}$ & $C_{s}$ & \\
& 0.000 & 0.024 & 0.087 & $C_{1}$ \\
\hline $\mathrm{F}$ & 0.000 & 0.019 & 0.064 & 0.052 \\
\hline $\mathrm{Cl}$ & 0.000 & 0.015 & 0.054 & 0.059 \\
\hline $\mathrm{Br}$ & 0.000 & 0.008 & 0.039 & 0.060 \\
\hline $\mathrm{I}$ & & & \\
\hline
\end{tabular}

- Optimized structures and relative energies (in $\mathrm{eV}$ ) for $\mathrm{Al}_{12} \mathrm{X}(\mathrm{X}=\mathrm{F}, \mathrm{Cl}, \mathrm{Br}$, and $\mathrm{I})$.

\begin{tabular}{|c|c|c|c|}
\hline & & \\
& $C_{s}$ & $C_{s}$ & \\
& 0.011 & 0.000 & $C_{1}$ \\
\hline $\mathrm{F}$ & 0.011 & 0.000 & 0.002 \\
\hline $\mathrm{Cl}$ & 0.004 & 0.000 & 0.016 \\
\hline $\mathrm{Br}$ & 0.000 & 0.006 & 0.020 \\
\hline $\mathrm{I}$ & & & \\
\hline
\end{tabular}

- Optimized structures and relative energies (in $\mathrm{eV}$ ) for $\mathrm{Al}_{13} \mathrm{X}(\mathrm{X}=\mathrm{F}, \mathrm{Cl}, \mathrm{Br}$, and $\mathrm{I})$.

\begin{tabular}{|c|c|c|c|c|}
\hline & $C_{s}$ & $C_{s}$ & $C_{2 v}$ & $C_{5 v}$ \\
\hline $\mathrm{F}$ & 0.000 & 0.204 & 0.574 & 0.528 \\
\hline $\mathrm{Cl}$ & 0.000 & 0.192 & 0.444 & 0.471 \\
\hline $\mathrm{Br}$ & 0.000 & 0.178 & 0.325 & 0.445 \\
\hline I & 0.000 & 0.153 & 0.170 & 0.397 \\
\hline
\end{tabular}


- Optimized structures and relative energies (in $\mathrm{eV}$ ) for $\mathrm{Al}_{14} \mathrm{X}(\mathrm{X}=\mathrm{F}, \mathrm{Cl}, \mathrm{Br}$, and $\mathrm{I})$.

\begin{tabular}{|c|c|c|c|}
\hline & & \\
& & $C_{s}$ & \\
& $C_{2 v}$ & \\
\hline $\mathrm{F}$ & 0.000 & 0.040 & $C_{s}$ \\
\hline $\mathrm{Cl}$ & 0.000 & 0.065 & 0.128 \\
\hline $\mathrm{Br}$ & 0.000 & 0.065 & 0.143 \\
\hline $\mathrm{I}$ & 0.000 & 0.153 & 0.143 \\
\hline
\end{tabular}

- Optimized structures and relative energies (in $\mathrm{eV}$ ) for $\mathrm{Al}_{15} \mathrm{X}(\mathrm{X}=\mathrm{F}, \mathrm{Cl}, \mathrm{Br}$, and I).

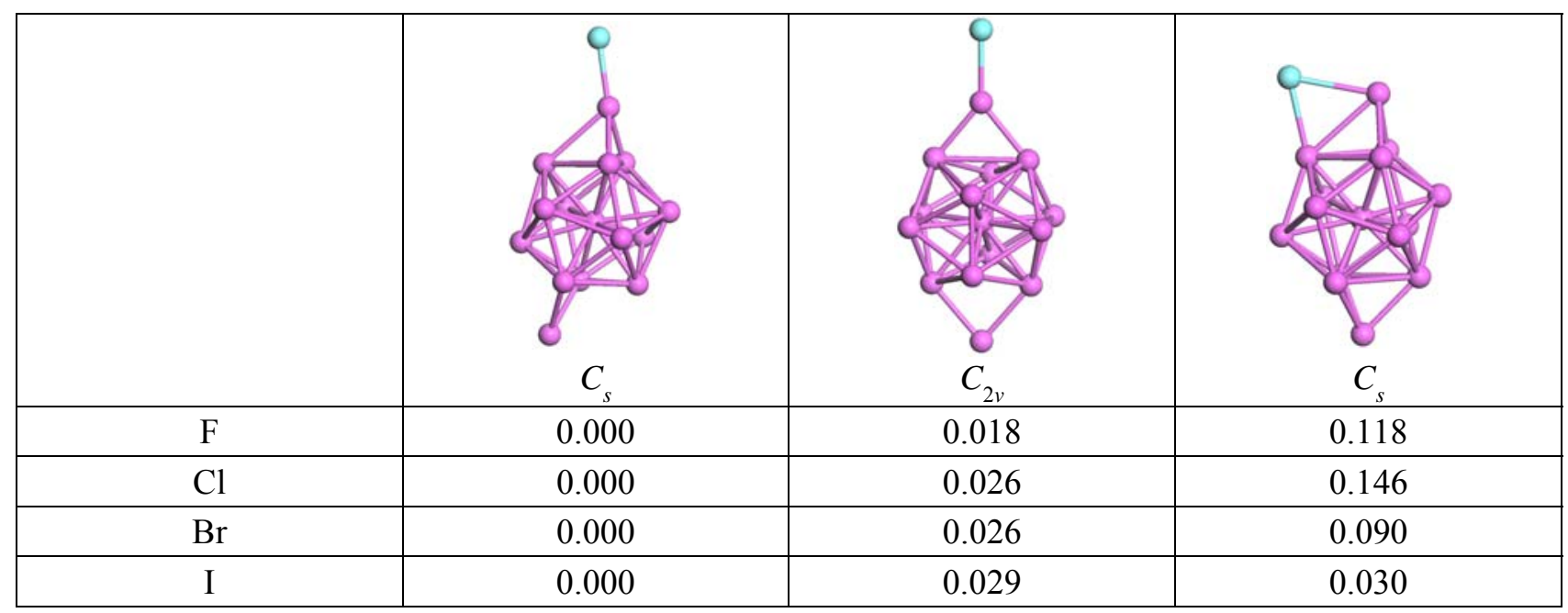


Figure S4. Optimized structures and relative energies (in eV) for $\mathrm{Al}_{11} \mathrm{X}_{2}(\mathrm{X}=\mathrm{F}, \mathrm{Cl}, \mathrm{Br}$, and I).

\begin{tabular}{|c|c|c|c|c|}
\hline & & & \\
& $C_{1}$ & $C_{1}$ & $C_{1}$ & \\
& 0.000 & 0.040 & 0.007 & $C_{2 v}$ \\
\hline $\mathrm{F}$ & 0.000 & 0.056 & 0.064 & 0.128 \\
\hline $\mathrm{Cl}$ & 0.000 & 0.058 & 0.067 & 0.095 \\
\hline $\mathrm{Br}$ & 0.000 & 0.060 & 0.084 & 0.086 \\
\hline $\mathrm{I}$ & &
\end{tabular}

- Optimized structures and relative energies (in eV) for $\mathrm{Al}_{12} \mathrm{X}_{2}(\mathrm{X}=\mathrm{F}, \mathrm{Cl}, \mathrm{Br}$, and I).

\begin{tabular}{|c|c|c|c|c|}
\hline & $C_{s}$ & $C_{1}$ & $C_{1}$ & $C_{s}$ \\
\hline & 0.023 & 0.000 & 0.072 & 0.011 \\
\hline $\mathrm{F}$ & 0.023 & 0.000 & 0.070 & 0.177 \\
\hline $\mathrm{Cl}$ & 0.014 & 0.000 & 0.069 & 0.158 \\
\hline $\mathrm{Br}$ & 0.000 & 0.044 & 0.107 & 0.188 \\
\hline $\mathrm{I}$ & & & \\
\hline
\end{tabular}

- Optimized structures and relative energies (in eV) for $\mathrm{Al}_{13} \mathrm{X}_{2}(\mathrm{X}=\mathrm{F}, \mathrm{Cl}, \mathrm{Br}$, and $\mathrm{I})$.

\begin{tabular}{|c|c|c|c|}
\hline & \\
& & & \\
& $C_{2 h}$ & $C_{s}$ \\
\hline $\mathrm{F}$ & 0.000 & 0.146 & $C_{s}$ \\
\hline $\mathrm{Cl}$ & 0.000 & 0.190 & 0.200 \\
\hline $\mathrm{Br}$ & 0.000 & 0.211 & 0.236 \\
\hline $\mathrm{I}$ & 0.000 & 0.246 & 0.248 \\
\hline
\end{tabular}


- Optimized structures and relative energies (in eV) for $\mathrm{Al}_{14} \mathrm{X}_{2}(\mathrm{X}=\mathrm{F}, \mathrm{Cl}, \mathrm{Br}$, and $\mathrm{I}$ ).

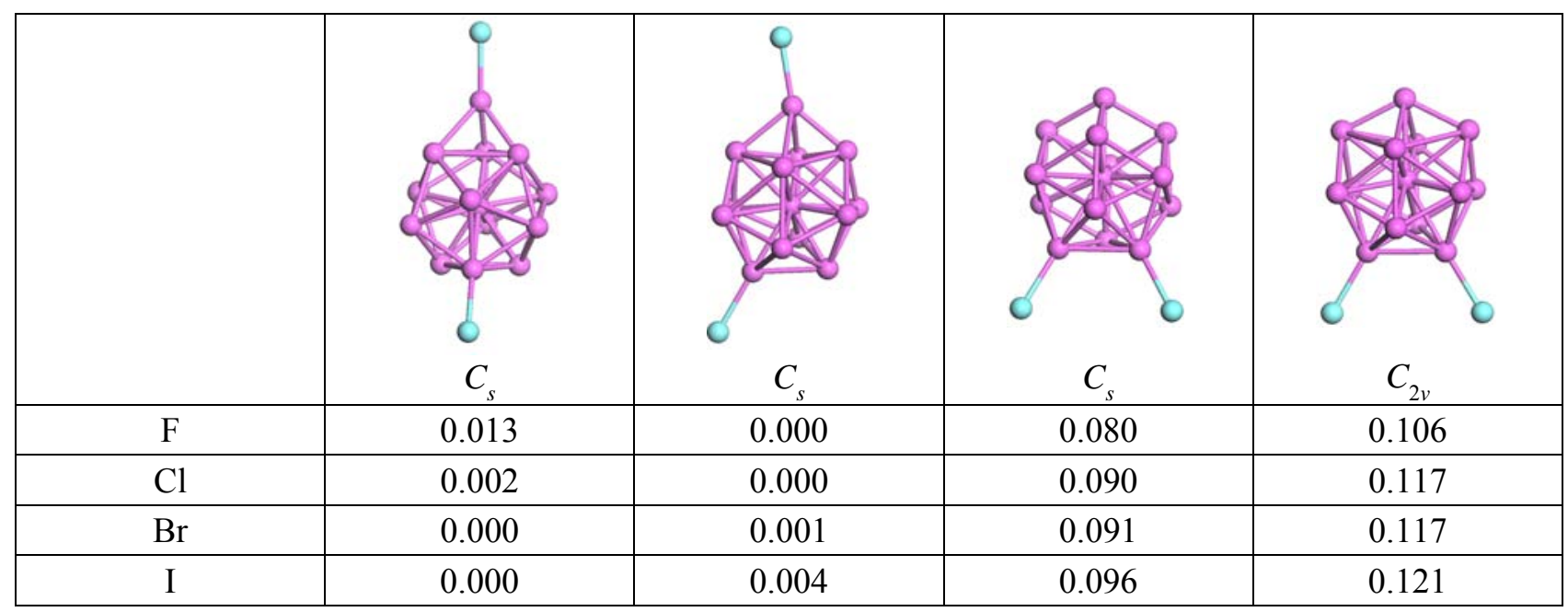

- Optimized structures and relative energies (in eV) for $\mathrm{Al}_{15} \mathrm{X}_{2}(\mathrm{X}=\mathrm{F}, \mathrm{Cl}, \mathrm{Br}$, and I).

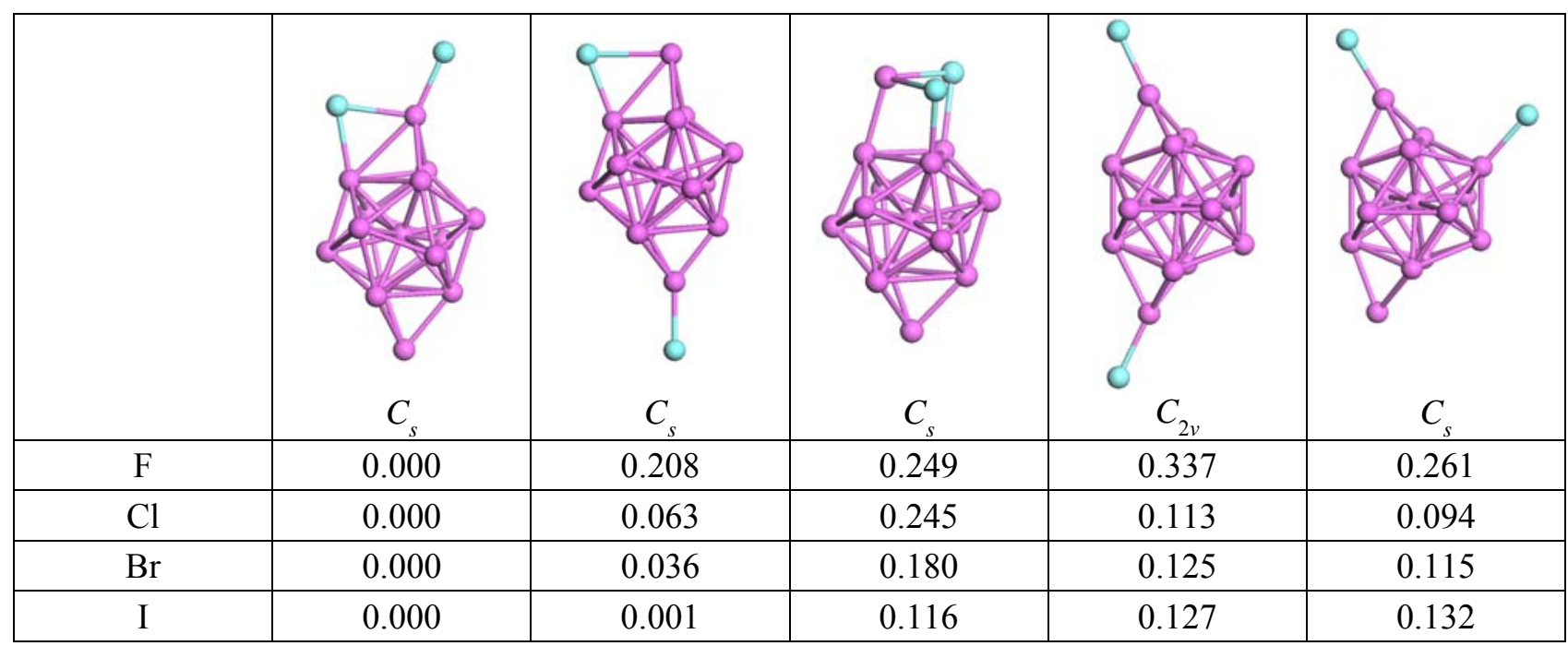


Table S1. (a) Charge $(Q)$ and electronegativity $(\eta)$ of $\mathrm{MX}\left(\mathrm{M}=\mathrm{Al}, \mathrm{Al}_{11}-\mathrm{Al}_{15}\right.$, Halogen atoms, $\left.\mathrm{X}=\mathrm{F}, \mathrm{Cl}, \mathrm{Br}, \mathrm{I}\right)$.

\begin{tabular}{cccccc}
\hline \multirow{2}{*}{$\mathrm{M}$} & $\eta(\mathrm{M})$ & \multicolumn{5}{c}{$Q(\mathrm{M})$} \\
\cline { 3 - 6 } & & $\mathrm{MF}$ & $\mathrm{MCl}$ & $\mathrm{MBr}$ & $\mathrm{MI}$ \\
\hline $\mathrm{Al}$ & 3.14 & 0.80 & 0.68 & 0.63 & 0.55 \\
$\mathrm{Al}_{11}$ & 4.36 & 0.69 & 0.46 & 0.37 & 0.24 \\
$\mathrm{Al}_{12}$ & 4.31 & 0.70 & 0.47 & 0.38 & 0.26 \\
$\mathrm{Al}_{13}$ & 4.66 & 0.70 & 0.48 & 0.39 & 0.26 \\
$\mathrm{Al}_{14}$ & 4.08 & 0.70 & 0.47 & 0.38 & 0.25 \\
$\mathrm{Al}_{15}$ & 4.18 & 0.71 & 0.48 & 0.39 & 0.26 \\
$\mathrm{~F}$ & 10.66 & 0.00 & -0.31 & -0.39 & -0.48 \\
$\mathrm{Cl}$ & 8.32 & 0.31 & 0.00 & -0.10 & -0.22 \\
$\mathrm{Br}$ & 7.60 & 0.39 & 0.10 & 0.00 & -0.12 \\
$\mathrm{I}$ & 6.87 & 0.48 & 0.22 & 0.12 & 0.00 \\
\hline
\end{tabular}

(b) Charge $(Q)$ and electronegativity $(\eta)$ of $\mathrm{MX}_{2}\left(\mathrm{M}=\mathrm{Al}, \mathrm{Al}_{11}-\mathrm{Al}_{15}\right.$, Si, Alkaline earth atoms, $\left.\mathrm{X}=\mathrm{F}, \mathrm{Cl}, \mathrm{Br}, \mathrm{I}\right)$.

\begin{tabular}{cccccc}
\hline \multirow{2}{*}{$\mathrm{M}$} & $\eta(\mathrm{M})$ & \multicolumn{4}{c}{$Q(\mathrm{M})$} \\
\cline { 3 - 6 } & & $\mathrm{MF}_{2}$ & $\mathrm{MCl}_{2}$ & $\mathrm{MBr}_{2}$ & $\mathrm{MI}_{2}$ \\
\hline $\mathrm{Al}$ & 3.14 & 1.18 & 0.94 & 0.85 & 0.71 \\
$\mathrm{Al}_{11}$ & 4.36 & 1.39 & 0.91 & 0.73 & 0.46 \\
$\mathrm{Al}_{12}$ & 4.31 & 1.39 & 0.92 & 0.75 & 0.43 \\
$\mathrm{Al}_{13}$ & 4.66 & 1.40 & 0.91 & 0.71 & 0.41 \\
$\mathrm{Al}_{14}$ & 4.08 & 1.41 & 0.95 & 0.78 & 0.52 \\
$\mathrm{Al} 1_{15}$ & 4.18 & 1.43 & 0.91 & 0.72 & 0.41 \\
$\mathrm{Si}$ & 4.66 & 1.33 & 0.84 & 0.66 & 0.40 \\
$\mathrm{Be}$ & 4.23 & 1.68 & 1.41 & 1.29 & 1.13 \\
$\mathrm{Mg}$ & 3.59 & 1.76 & 1.60 & 1.50 & 1.37 \\
$\mathrm{Ca}$ & 3.00 & 1.73 & 1.71 & 1.67 & 1.60 \\
$\mathrm{Sr}$ & 2.89 & 1.82 & 1.73 & 1.70 & 1.65 \\
$\mathrm{Ba}$ & 2.42 & 1.80 & 1.73 & 1.69 & 1.64 \\
\hline
\end{tabular}


(c) Optimized structures of MX ( $\mathrm{M}=\mathrm{Al}$, Halogen atoms, $\mathrm{X}=\mathrm{F}, \mathrm{Cl}, \mathrm{Br}, \mathrm{I})$.

\begin{tabular}{ccccc}
\hline \multirow{2}{*}{$\mathrm{M}$} & \multicolumn{5}{c}{ Bond length $(\AA)$} \\
\cline { 2 - 5 } & $\mathrm{MF}$ & $\mathrm{MCl}$ & $\mathrm{MBr}$ & $\mathrm{MI}$ \\
\hline $\mathrm{Al}$ & 1.70 & 2.17 & 2.34 & 2.57 \\
$\mathrm{~F}$ & 1.41 & 1.66 & 1.79 & 1.94 \\
$\mathrm{Cl}$ & - & 2.02 & 2.17 & 2.35 \\
$\mathrm{Br}$ & - & - & 2.31 & 2.50 \\
$\mathrm{I}$ & - & - & - & 2.69 \\
\hline
\end{tabular}

(d) Optimized structures of $\mathrm{MX}_{2}(\mathrm{M}=\mathrm{Al}, \mathrm{Si}$, Alkaline earth atoms, $\mathrm{X}=\mathrm{F}, \mathrm{Cl}, \mathrm{Br}, \mathrm{I})$.

\begin{tabular}{ccccccccc}
\hline & \multicolumn{9}{c}{ Bond length $(\AA)$} \\
\cline { 2 - 8 } $\mathrm{M}$ & $\mathrm{MF}_{2}$ & $\mathrm{MCl}_{2}$ & $\mathrm{MBr}_{2}$ & $\mathrm{MI}_{2}$ & $\mathrm{MF}_{2}$ & $\mathrm{MCl}_{2}$ & $\mathrm{MBr}_{2}$ & $\mathrm{MI}_{2}$ \\
\hline $\mathrm{Al}$ & 1.68 & 2.13 & 2.29 & 2.52 & 120.6 & 119.6 & 120.4 & 121.0 \\
$\mathrm{Si}$ & 1.63 & 2.11 & 2.28 & 2.50 & 100.7 & 101.7 & 102.6 & 103.6 \\
$\mathrm{Be}$ & 1.39 & 1.80 & 1.96 & 2.17 & 180.0 & 180.0 & 180.0 & 180.0 \\
$\mathrm{Mg}$ & 1.77 & 2.19 & 2.33 & 2.54 & 180.0 & 180.0 & 180.0 & 180.0 \\
$\mathrm{Ca}$ & 1.99 & 2.46 & 2.61 & 2.83 & 134.3 & 161.7 & 180.0 & 180.0 \\
$\mathrm{Sr}$ & 2.13 & 2.60 & 2.76 & 2.99 & 127.8 & 137.9 & 146.9 & 156.1 \\
$\mathrm{Ba}$ & 2.24 & 2.74 & 2.90 & 3.13 & 120.2 & 126.5 & 128.1 & 131.0 \\
\hline
\end{tabular}
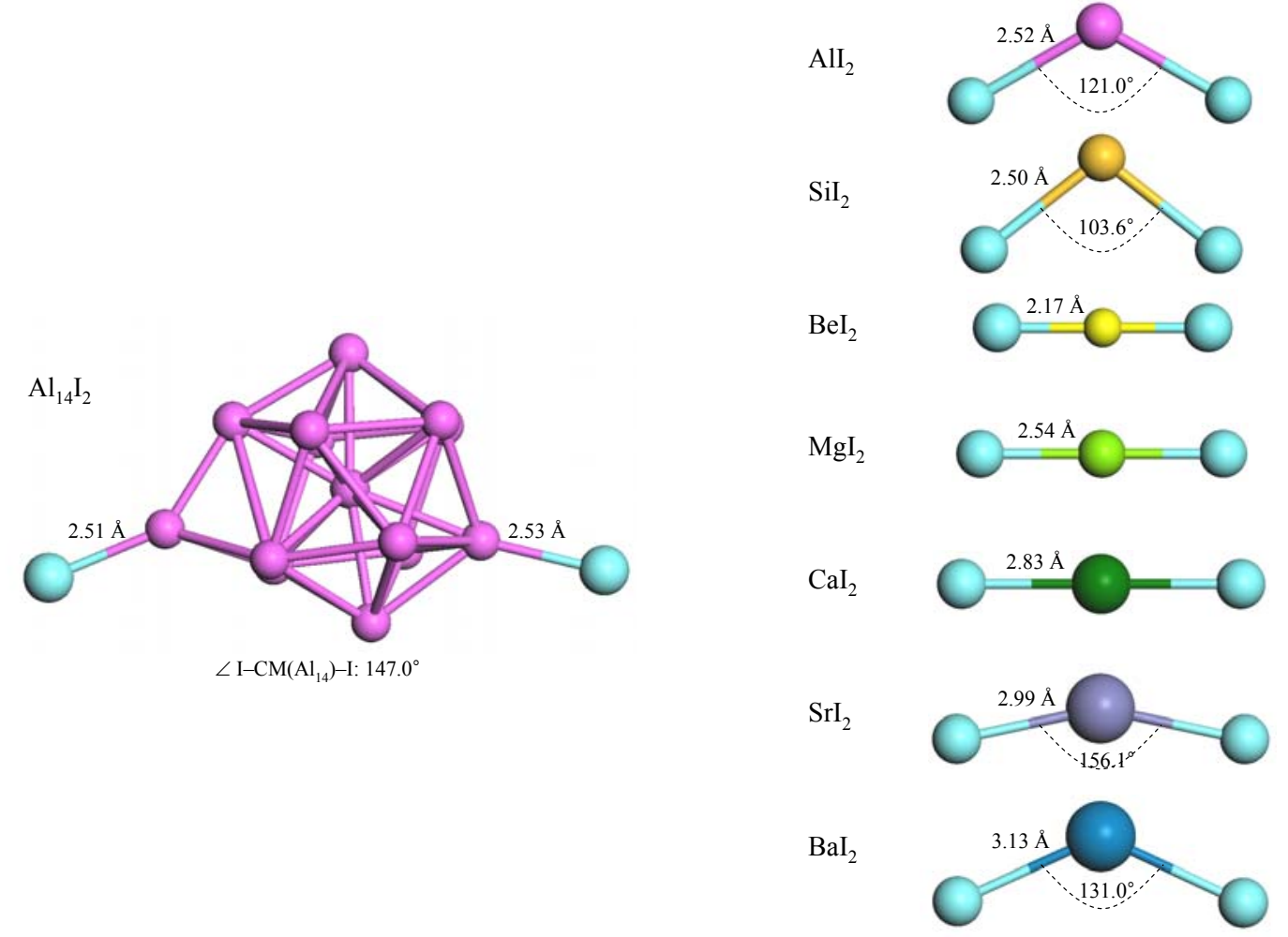
Figure S5. Optimized structures and relative energies (in eV) for $\mathrm{Al}_{n} \mathrm{I}_{2}^{-}(n=11-15)$.

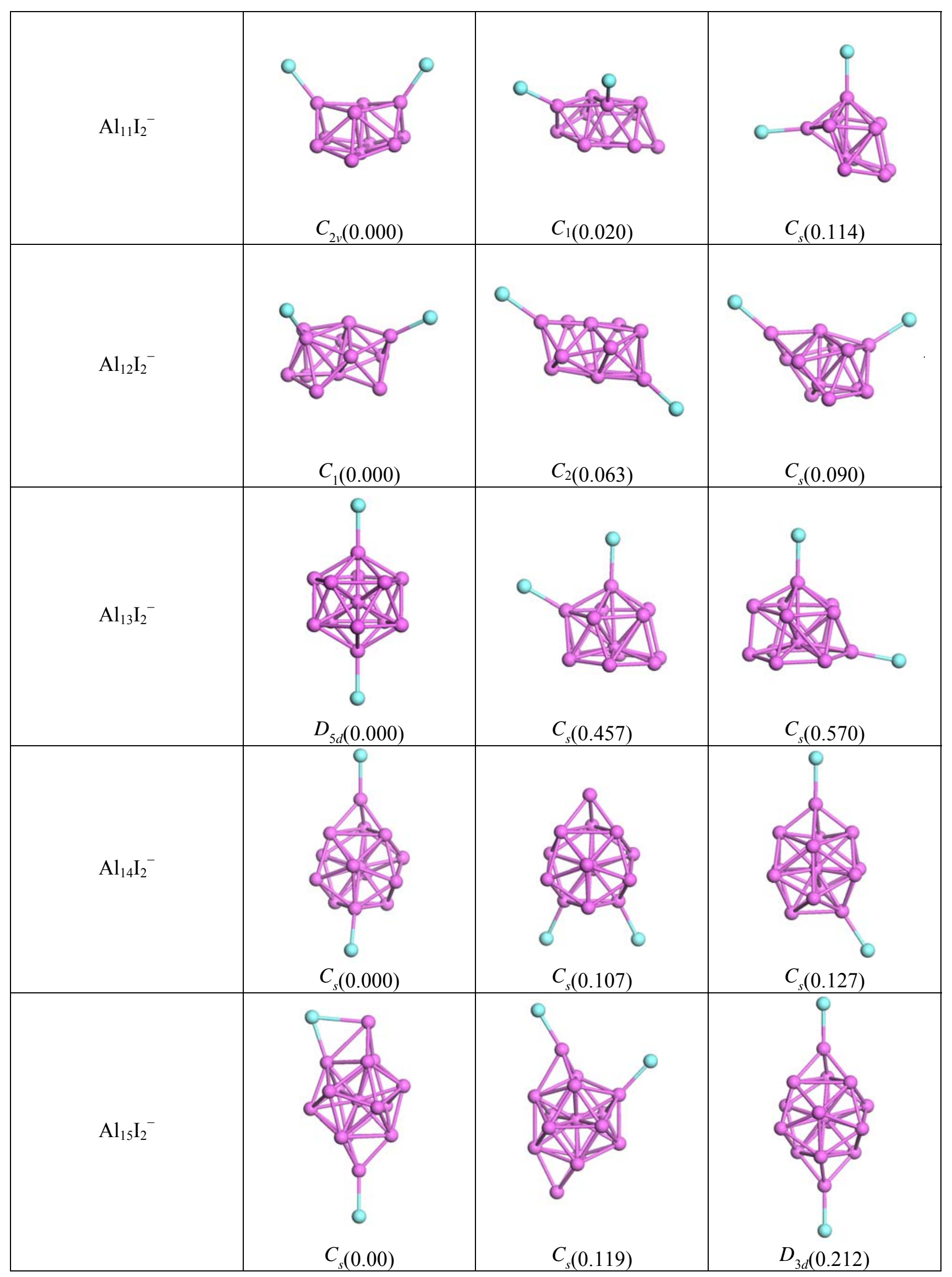


Figure S6. Optimized structures and relative energies (in eV) for $\mathrm{Al}_{n} \mathrm{I}_{3}^{-}(n=11-15)$.




Table S2. Charge distribution $Q\left(\mathrm{Al}_{n}\right)$ for $\mathrm{Al}_{n} \mathrm{I}_{2}{ }^{-}$and $\mathrm{Al}_{n} \mathrm{I}_{3}{ }^{-}(n=11-15)$.

\begin{tabular}{ccc}
\hline$n$ & $\mathrm{Al}_{\mathrm{n}} \mathrm{I}_{2}^{-}$ & $\mathrm{Al}_{\mathrm{n}} \mathrm{I}_{3}^{-}$ \\
\hline 11 & -0.36 & -0.04 \\
12 & -0.33 & -0.01 \\
13 & -0.38 & -0.10 \\
14 & -0.32 & -0.01 \\
15 & -0.42 & -0.03 \\
\hline
\end{tabular}

Figure S7. HOMO-LUMO gaps for $\mathrm{Al}_{n} \mathrm{I}_{2}^{-}$and $\mathrm{Al}_{n} \mathrm{I}_{3}^{-}(n=11-15)$.

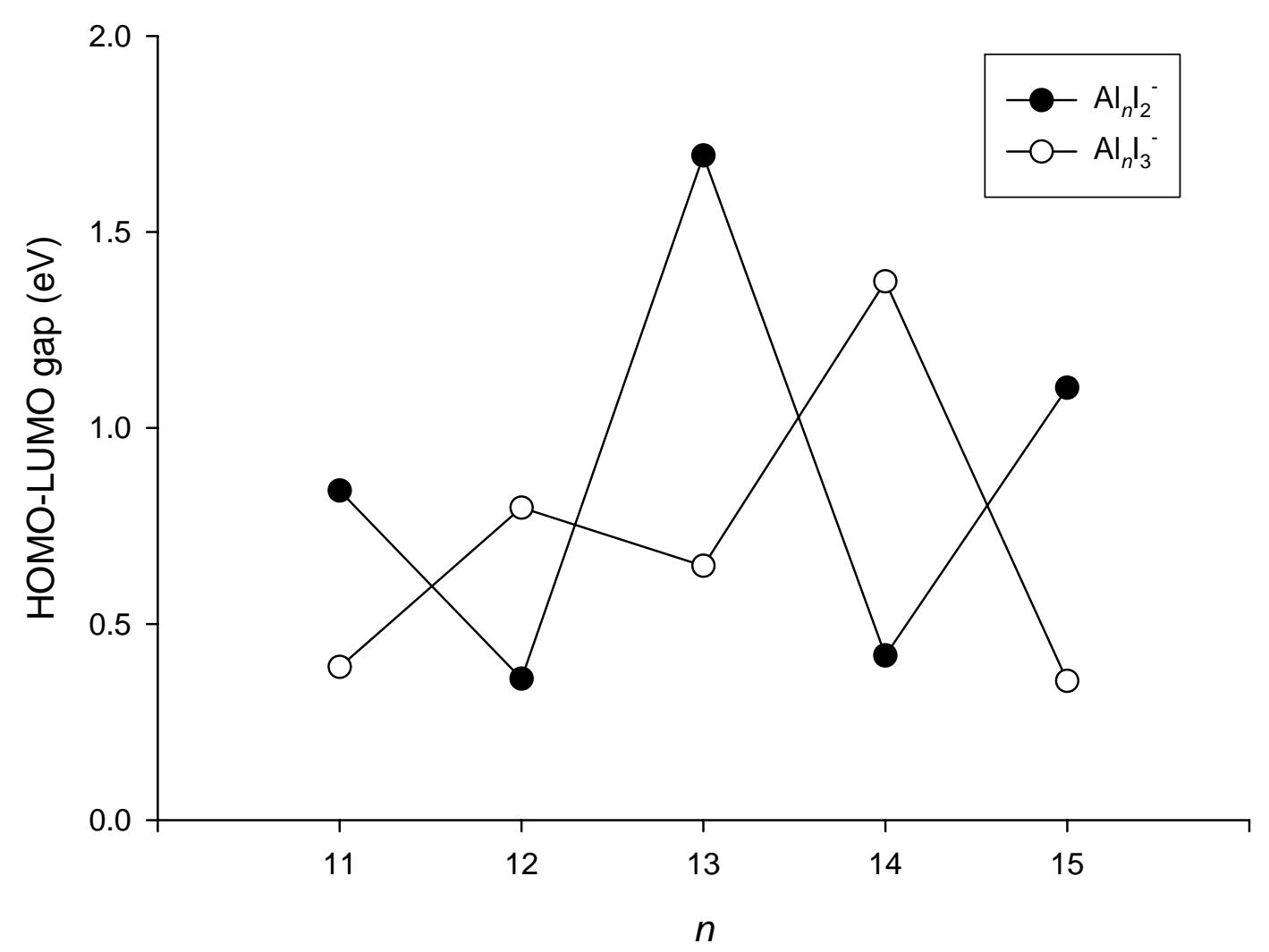


Figure S8. Molecular orbital (MO) diagram for $\mathrm{Al}_{13}{ }^{-}$and $\mathrm{Al}_{13} \mathrm{~K}^{-}$. Units are in $\mathrm{eV}$.

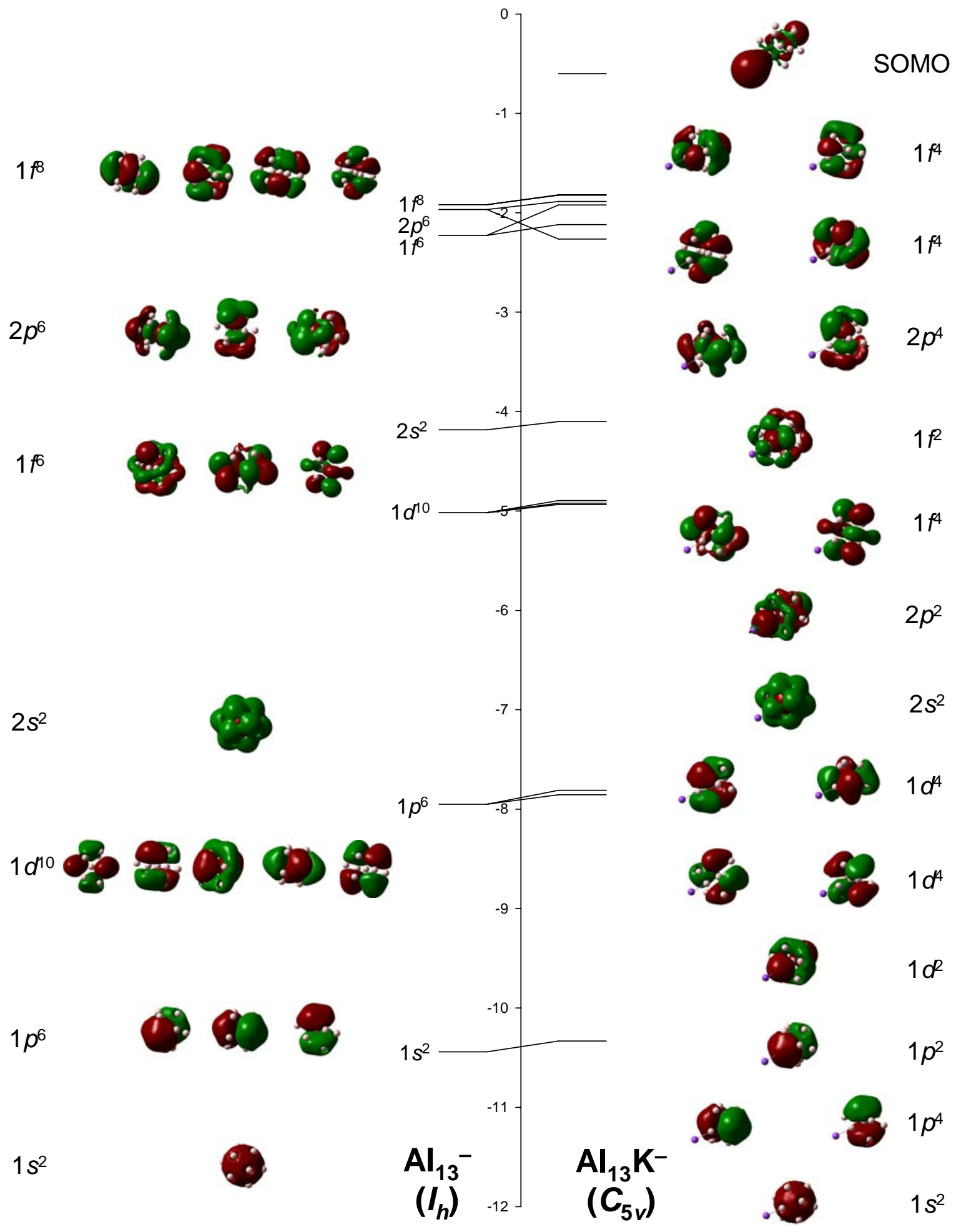


Figure S9. Molecular orbital (MO) diagram for $\mathrm{Al}_{15}{ }^{-}$and $\mathrm{Al}_{15} \mathrm{I}_{2}{ }^{-}$. Units are in $\mathrm{eV}$.

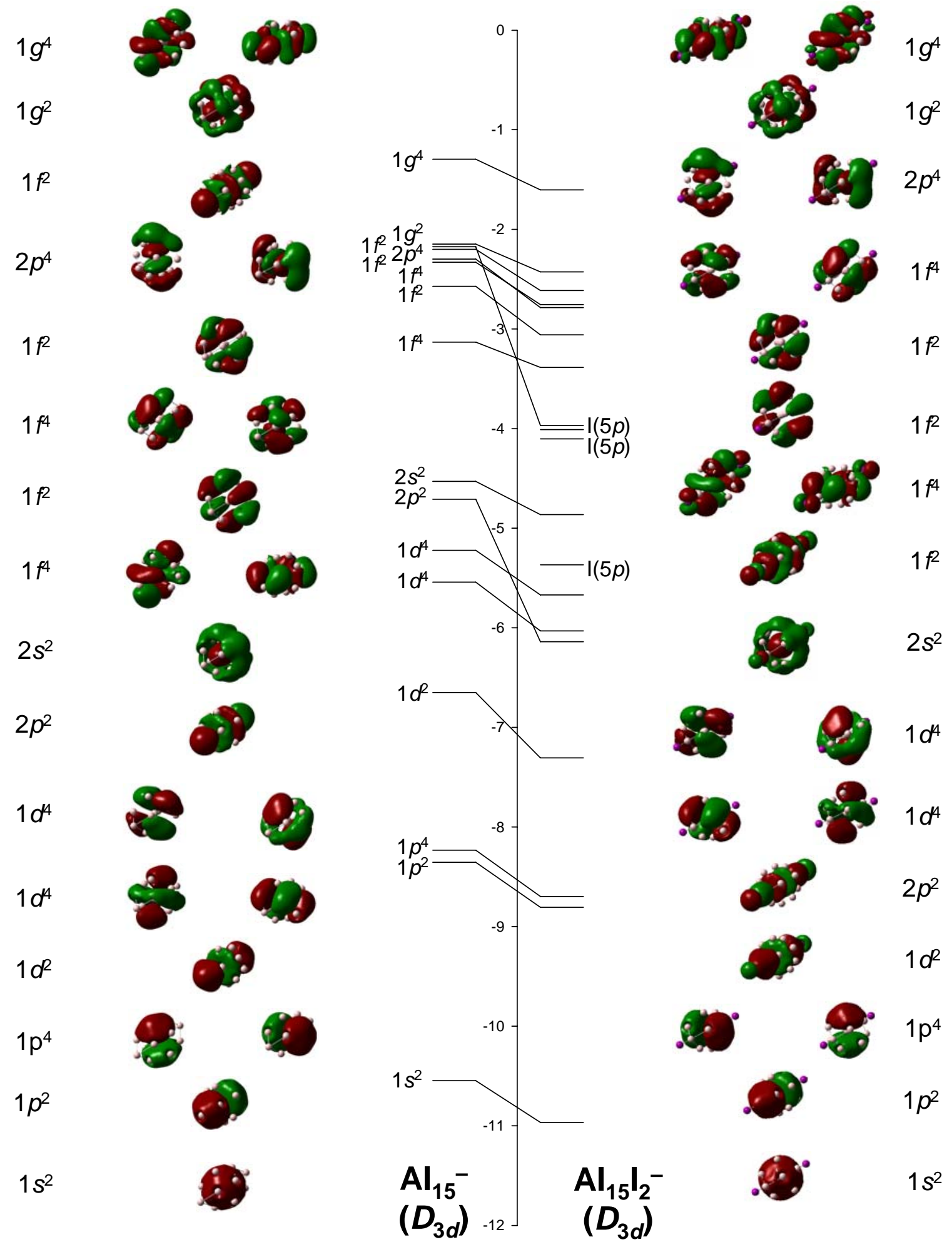


Table S3. (a) Absolute energies (in Hartrees) for $\mathrm{Al}_{n}{ }^{q}(n=11-15, q=-1,0,+1$, and +2$)$.

\begin{tabular}{|c|c|c|c|c|}
\hline$q$ & -1 & 0 & +1 & +2 \\
\hline $\mathrm{Al}_{11}$ & -2665.60520858 & -2665.50848620 & -2665.28481799 & -2664.93262705 \\
\hline $\mathrm{Al}_{12}$ & -2907.94751851 & -2907.85426739 & -2907.63102865 & -2907.28773356 \\
\hline $\mathrm{Al}_{13}$ & -3150.34556013 & -3150.22299354 & -3150.00278909 & -3149.64104748 \\
\hline $\mathrm{Al}_{14}$ & -3392.65146660 & -3392.56281536 & -3392.35128607 & -3392.03461113 \\
\hline $\mathrm{Al}_{15}$ & -3634.98683887 & -3634.88795703 & -3634.67964418 & -3634.34230279 \\
\hline
\end{tabular}

(b) Absolute energies (in Hartrees) for $\mathrm{Al}_{n} \mathrm{X}(n=11-15, \mathrm{X}=\mathrm{F}, \mathrm{Cl}, \mathrm{Br}$, and I).

\begin{tabular}{|c|c|c|c|c|}
\hline $\mathrm{X}$ & $\mathrm{F}$ & $\mathrm{Cl}$ & $\mathrm{Br}$ & $\mathrm{I}$ \\
\hline $\mathrm{Al}_{11}$ & -2765.38322678 & -3125.62496582 & -3082.44236270 & -2963.28204325 \\
\hline $\mathrm{Al}_{12}$ & -3007.72252544 & -3367.96421276 & -3324.78164620 & -3205.62159595 \\
\hline $\mathrm{Al}_{13}$ & -3250.09692144 & -3610.33902554 & -3567.15652412 & -3447.99640210 \\
\hline $\mathrm{Al}_{14}$ & -3492.44202282 & -3852.68321321 & -3809.50048990 & -3690.33990766 \\
\hline $\mathrm{Al}_{15}$ & -3734.77005697 & -4095.01154639 & -4051.82896794 & -3932.66851047 \\
\hline
\end{tabular}

(c) Absolute energies (in Hartrees) for $\mathrm{Al}_{n} \mathrm{X}_{2}(n=11-15, \mathrm{X}=\mathrm{F}, \mathrm{Cl}, \mathrm{Br}$, and I).

\begin{tabular}{|c|c|c|c|c|}
\hline $\mathrm{X}$ & $\mathrm{F}$ & $\mathrm{Cl}$ & $\mathrm{Br}$ & $\mathrm{I}$ \\
\hline $\mathrm{Al}_{11}$ & -2865.25503782 & -3585.73836296 & -3499.37332468 & -3261.05270292 \\
\hline $\mathrm{Al}_{12}$ & -3107.59887128 & -3828.08233605 & -3741.71679511 & -3503.39829480 \\
\hline $\mathrm{Al}_{13}$ & -3349.95421094 & -4070.43887683 & -3984.07453919 & -3745.75539482 \\
\hline $\mathrm{Al}_{14}$ & -3592.33015793 & -4312.81054292 & -4226.44477525 & -3988.12314778 \\
\hline $\mathrm{Al}_{15}$ & -3834.64297607 & -4555.11899297 & -4468.75457736 & -4230.43440481 \\
\hline
\end{tabular}

(d) Absolute energies (in Hartrees) for $\mathrm{Al}_{n} \mathrm{I}^{-}, \mathrm{Al}_{n} \mathrm{I}_{2}^{-}$, and $\mathrm{Al}_{n} \mathrm{I}_{3}{ }^{-}(n=11-15)$.

\begin{tabular}{|c|c|c|c|}
\hline & $\mathrm{Al}_{n} \mathrm{I}^{-}$ & $\mathrm{Al}_{n} \mathrm{I}_{2}^{-}$ & $\mathrm{Al}_{n} \mathrm{I}_{3}{ }^{-}$ \\
\hline $\mathrm{Al}_{11}$ & -2963.38113063 & -3261.16172265 & -3558.93362619 \\
\hline $\mathrm{Al}_{12}$ & -3205.72798129 & -3503.49999744 & -3801.28114362 \\
\hline $\mathrm{Al}_{13}$ & -3448.09947707 & -3745.88466867 & -4043.63684835 \\
\hline $\mathrm{Al}_{14}$ & -3690.43497601 & -3988.21492559 & -4286.00128733 \\
\hline $\mathrm{Al}_{15}$ & -3932.76616642 & -4230.54664927 & -4528.30784632 \\
\hline
\end{tabular}


(e) Cartesian coordinates.

- $\mathrm{Al}_{n}{ }^{q}(n=11-15, q=-1,0,+1$, and +2$)$
Al $\quad 0.000000000 \quad 1.335536000 \quad-1.289565000$

Al $\quad 2.388851000 \quad-0.000000000 \quad-1.519271000$

$\begin{array}{lrrr}\mathbf{A l}_{11}{ }^{2+} & & & \\ \mathrm{Al} & -1.818038000 & 2.283250000 & 0.163780000 \\ \mathrm{Al} & 0.000000000 & 2.299331000 & -1.812316000 \\ \mathrm{Al} & 1.818038000 & 2.283250000 & 0.163780000 \\ \mathrm{Al} & 0.000000000 & 2.562390000 & 2.013352000 \\ \mathrm{Al} & 0.000000000 & -0.000000000 & 1.202435000 \\ \mathrm{Al} & 1.422855000 & -0.000000000 & -1.129813000 \\ \mathrm{Al} & 1.818038000 & -2.283250000 & 0.163780000 \\ \mathrm{Al} & -0.000000000 & -2.562390000 & 2.013352000 \\ \mathrm{Al} & -1.818038000 & -2.283250000 & 0.163780000 \\ \mathrm{Al} & -0.000000000 & -2.299331000 & -1.812316000 \\ \mathrm{Al} & -1.422855000 & 0.000000000 & -1.129813000\end{array}$

$\mathrm{Al}_{11}^{+}$

Al $\quad-1.693423000 \quad-1.611182000 \quad-1.375467000$

Al $\quad 2.986774000 \quad 0.114473000 \quad-0.872896000$

Al $\quad 1.704099000 \quad 2.066846000 \quad 0.452714000$

Al $\quad-2.419529000 \quad 1.072738000 \quad-0.837784000$

Al $\quad-2.734814000 \quad-0.894628000 \quad 0.991801000$

Al $\quad-0.267111000 \quad-1.973682000 \quad 0.883792000$

Al $\quad \begin{array}{llll}1.731871000 & -0.363793000 & 1.483679000\end{array}$

Al $\quad-0.626995000 \quad 2.866504000 \quad-0.422382000$

Al $\quad-0.729502000 \quad 0.744229000 \quad 1.415079000$

Al $\quad \begin{array}{llll}1.839086000 & -2.195035000 & -0.622331000\end{array}$

Al $\quad 0.209543000 \quad 0.173530000 \quad-1.096204000$

$\mathrm{Al}_{11}$

$\begin{array}{lrrr}\text { Al } & 2.217439000 & 1.518963000 & 0.606695000 \\ \text { Al } & -2.217439000 & 1.518963000 & 0.606695000 \\ \text { Al } & 2.217439000 & -1.518963000 & 0.606695000 \\ \text { Al } & 0.000000000 & 0.000000000 & 1.107927000 \\ \text { Al } & 0.000000000 & 2.844191000 & 1.041483000 \\ \text { Al } & -0.000000000 & -2.844191000 & 1.041483000 \\ \text { Al } & -2.217439000 & -1.518963000 & 0.606695000 \\ \text { Al } & -2.388851000 & 0.000000000 & -1.519271000 \\ \text { Al } & 0.000000000 & -1.335536000 & -1.289565000\end{array}$

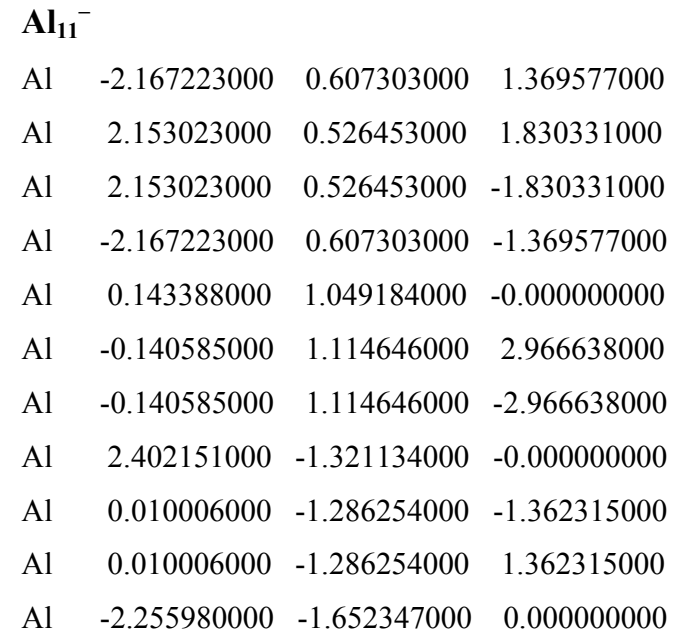
$\mathrm{Al}_{12}^{+}$
Al $\quad 1.695230000 \quad 0.197166000 \quad 2.189900000$
Al $\quad 3.030119000 \quad 0.455004000 \quad 0.000000000$
Al $\quad-0.393398000 \quad 1.694375000 \quad-2.678974000$
Al $\quad-2.265348000 \quad 0.450252000 \quad-1.340723000$
Al $\quad 0.084012000 \quad 0.923820000 \quad 0.000000000$
Al $\quad-0.393398000 \quad 1.694375000 \quad 2.678974000$
Al $\quad 1.136366000 \quad-1.540180000 \quad 0.000000000$ 
Al $\quad \begin{array}{llll}1.695230000 & 0.197166000 & -2.189900000\end{array}$

Al $\quad-0.393398000 \quad-1.346823000 \quad-2.234177000$

Al $\quad-1.536669000 \quad-1.828583000 \quad 0.000000000$

Al $\quad-2.265348000 \quad 0.450252000 \quad 1.340723000$

Al $\quad-0.393398000 \quad-1.346823000 \quad 2.234177000$
Al $\quad 1.051321000 \quad 1.015701000 \quad-2.243475000$

Al $\quad \begin{array}{llll}1.913666000 & -1.599412000 & -1.378894000\end{array}$

Al $\quad-0.359199000 \quad-1.226663000 \quad-2.673509000$

Al $\quad 2.604502000 \quad 0.641613000 \quad-0.000000000$

Al $\quad-0.144767000 \quad-0.497431000 \quad 0.000000000$

$\begin{array}{lrrr}\mathrm{Al}_{\mathbf{1 2}} & & & \\ \mathrm{Al} & 1.822441000 & 0.074787000 & 2.170025000 \\ \mathrm{Al} & 2.760163000 & 1.139268000 & 0.000000000 \\ \mathrm{Al} & 1.281289000 & -1.435235000 & 0.000000000 \\ \mathrm{Al} & -0.356984000 & -1.330204000 & 2.167086000 \\ \mathrm{Al} & -0.356984000 & 1.565504000 & 2.601746000 \\ \mathrm{Al} & -1.459000000 & -2.003474000 & 0.000000000 \\ \mathrm{Al} & 1.822441000 & 0.074787000 & -2.170025000 \\ \mathrm{Al} & -0.356984000 & -1.330204000 & -2.167086000 \\ \mathrm{Al} & -2.380220000 & 0.411717000 & -1.317689000 \\ \mathrm{Al} & -0.038955000 & 0.855830000 & 0.000000000 \\ \mathrm{Al} & -0.356984000 & 1.565504000 & -2.601746000 \\ \mathrm{Al} & -2.380220000 & 0.411717000 & 1.317689000\end{array}$
$\mathrm{Al}_{13}{ }^{+}$
Al $\quad 1.317946000 \quad 0.129209000 \quad-2.624804000$
Al $\quad 1.189347000 \quad 2.388420000 \quad-1.308050000$
Al $\quad-1.352227000 \quad 1.763219000 \quad-1.406948000$
Al $\quad-1.103185000 \quad-0.866641000 \quad-2.200568000$
Al $\quad \begin{array}{llll}1.393807000 & -2.155495000 & -1.320316000\end{array}$
Al $\quad-2.356278000 \quad-0.171432000 \quad 0.000000000$
Al $\quad 1.189347000 \quad 2.388420000 \quad 1.308050000$
Al $\quad-1.352227000 \quad 1.763219000 \quad 1.406948000$
Al $\quad-1.103185000 \quad-0.866641000 \quad 2.200568000$
Al $\quad 1.393807000 \quad-2.155495000 \quad 1.320316000$
Al $\quad 1.317946000 \quad 0.129209000 \quad 2.624804000$
Al $\quad-0.994399000 \quad-2.419193000 \quad 0.000000000$
Al $\quad 0.459303000 \quad 0.073201000 \quad 0.000000000$
$\mathbf{A l}_{13}$
Al $\quad-1.479953000 \quad 0.854451000 \quad 2.035408000$
Al $\quad 1.479953000 \quad 0.854451000 \quad 2.035408000$
Al $\quad 0.000000000 \quad 2.648835000 \quad 0.558478000$
Al $\quad 0.000000000 \quad 0.000000000 \quad 0.000000000$
Al $\quad-2.293958000 \quad-1.324418000 \quad 0.558478000$
Al $\quad 0.000000000 \quad-1.708902000 \quad 2.035408000$
Al $\quad 2.293958000 \quad-1.324417000 \quad 0.558478000$
Al $\quad 2.293958000 \quad 1.324418000 \quad-0.558478000$
Al $\quad 0.000000000 \quad 1.708902000 \quad-2.035408000$
Al $\quad-2.293958000 \quad 1.324418000 \quad-0.558478000$
Al $\quad 0.000000000 \quad-2.648835000 \quad-0.558478000$
Al $\quad \begin{array}{llll}1.479953000 & -0.854451000 & -2.035408000\end{array}$
Al $\quad-1.479953000 \quad-0.854451000 \quad-2.035408000$ 


$\begin{array}{lrrr}\text { Al } & 0.000000000 & -2.388429000 & -1.194214000 \\ \text { Al } & 0.000000000 & 0.000000000 & -2.670345000 \\ \text { Al } & 1.403883000 & 1.932280000 & -1.194214000 \\ \text { Al } & 2.271531000 & 0.738065000 & 1.194214000 \\ \text { Al } & 2.271531000 & -0.738065000 & -1.194214000 \\ \text { Al } & -1.403883000 & 1.932280000 & -1.194214000 \\ \text { Al } & 0.000000000 & 0.000000000 & 0.000000000\end{array}$

$\begin{array}{lrrr}\mathbf{A l}_{\mathbf{1 4}}{ }^{2+} & & & \\ \mathrm{Al} & -2.048698000 & 1.182816000 & 1.673857000 \\ \mathrm{Al} & 2.048698000 & 1.182816000 & 1.673857000 \\ \mathrm{Al} & 0.000000000 & 2.599848000 & 0.432931000 \\ \mathrm{Al} & 0.000000000 & 0.000000000 & 0.073369000 \\ \mathrm{Al} & -2.251534000 & -1.299924000 & 0.432931000 \\ \mathrm{Al} & 0.000000000 & -2.365633000 & 1.673857000 \\ \mathrm{Al} & 2.251534000 & -1.299924000 & 0.432931000 \\ \mathrm{Al} & 2.328840000 & 1.344556000 & -0.897179000 \\ \mathrm{Al} & 0.000000000 & 1.602707000 & -2.176168000 \\ \mathrm{Al} & -2.328840000 & 1.344556000 & -0.897179000 \\ \mathrm{Al} & 0.000000000 & -2.689113000 & -0.897179000 \\ \mathrm{Al} & 1.387985000 & -0.801353000 & -2.176168000 \\ \mathrm{Al} & -1.387985000 & -0.801354000 & -2.176168000 \\ \mathrm{Al} & 0.000000000 & 0.000000000 & 2.826307000\end{array}$

\footnotetext{
$\mathrm{Al}_{14}$

Al $\quad 0.000000000 \quad 2.652266000 \quad 0.176658000$

Al $\quad 1.429270000 \quad 0.825189000 \quad 1.746144000$
}

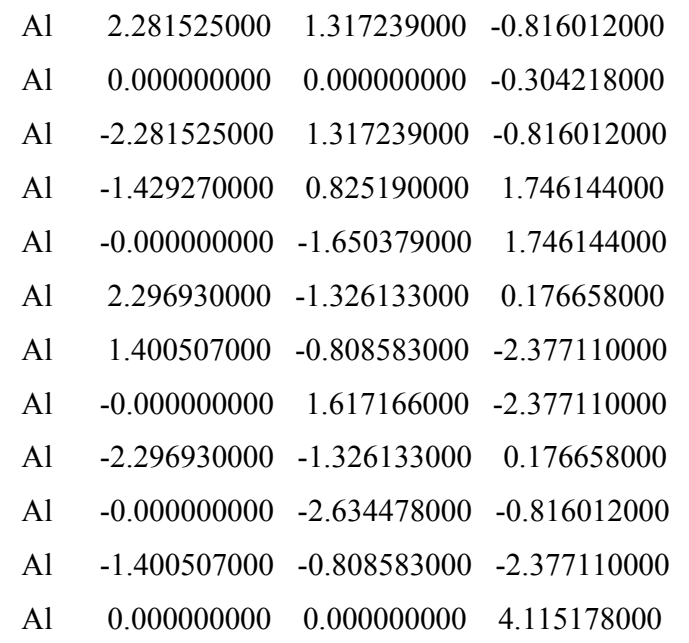

$\begin{array}{lrrr}\mathrm{Al}_{15}{ }^{2+} & & & \\ \mathrm{Al} & -1.131459000 & 0.800480000 & 2.255808000 \\ \mathrm{Al} & -0.833379000 & -1.852191000 & 1.482122000 \\ \mathrm{Al} & 1.413019000 & -0.669922000 & 2.327412000 \\ \mathrm{Al} & 0.130449000 & 0.358632000 & 0.000000000 \\ \mathrm{Al} & -2.076902000 & 2.132926000 & 0.000000000 \\ \mathrm{Al} & -2.554266000 & -0.417453000 & -0.000000000 \\ \mathrm{Al} & -0.833379000 & -1.852191000 & -1.482122000 \\ \mathrm{Al} & 1.522015000 & -2.008476000 & -0.000000000 \\ \mathrm{Al} & 2.760424000 & 0.465233000 & 0.000000000 \\ \mathrm{Al} & 1.413019000 & 1.902467000 & 2.018346000 \\ \mathrm{Al} & -1.131459000 & 0.800480000 & -2.255808000 \\ \mathrm{Al} & 1.413019000 & -0.669922000 & -2.327412000 \\ \mathrm{Al} & 1.413019000 & 1.902467000 & -2.018346000 \\ \mathrm{Al} & -1.866181000 & -4.043918000 & -0.000000000\end{array}$




$\begin{array}{lrrr}\mathrm{Al}_{15}{ }^{+} & & & \\ \mathrm{Al} & -0.000000000 & 2.205141000 & -1.524776000 \\ \mathrm{Al} & 1.919454000 & -0.000000000 & -2.091721000 \\ \mathrm{Al} & 2.249493000 & 1.548474000 & 0.017031000 \\ \mathrm{Al} & 0.000000000 & 2.298980000 & 1.329385000 \\ \mathrm{Al} & -2.249493000 & 1.548474000 & 0.017031000 \\ \mathrm{Al} & 1.642859000 & -0.000000000 & 2.115589000 \\ \mathrm{Al} & -0.000000000 & -0.000000000 & -3.732221000 \\ \mathrm{Al} & 2.249493000 & -1.548474000 & 0.017031000 \\ \mathrm{Al} & -0.000000000 & -2.298980000 & 1.329385000 \\ \mathrm{Al} & -2.249493000 & -1.548474000 & 0.017031000 \\ \mathrm{Al} & -0.000000000 & -2.205141000 & -1.524776000 \\ \mathrm{Al} & -1.642859000 & 0.000000000 & 2.115589000 \\ \mathrm{Al} & -0.000000000 & -0.000000000 & -0.105605000 \\ \mathrm{Al} & 0.000000000 & 0.000000000 & 4.112746000 \\ \mathrm{Al} & -1.919454000 & 0.000000000 & -2.091721000\end{array}$

$\begin{array}{lrrr}\mathbf{A l}_{15} & & & \\ \mathrm{Al} & 3.103508000 & -2.438709000 & 0.000000000 \\ \mathrm{Al} & 1.187820000 & -1.861432000 & 1.645124000 \\ \mathrm{Al} & -1.187820000 & -0.802397000 & 2.269871000 \\ \mathrm{Al} & 1.187820000 & 0.802397000 & 2.269871000 \\ \mathrm{Al} & 2.676082000 & 0.265456000 & 0.000000000 \\ \mathrm{Al} & -1.187820000 & 1.861432000 & 1.645124000 \\ \mathrm{Al} & 1.187820000 & -1.861432000 & -1.645124000 \\ \mathrm{Al} & -2.676082000 & -0.265456000 & 0.000000000 \\ \mathrm{Al} & -1.187820000 & 1.861432000 & -1.645124000 \\ \mathrm{Al} & 1.187820000 & 0.802397000 & -2.269871000 \\ \mathrm{Al} & -1.187820000 & -0.802397000 & -2.269871000 \\ \mathrm{Al} & 1.027818000 & 2.470692000 & -0.000000000 \\ \mathrm{Al} & 0.000000000 & 0.000000000 & 0.000000000 \\ \mathrm{Al} & -1.027818000 & -2.470692000 & -0.000000000 \\ \mathrm{Al} & -3.103508000 & 2.438709000 & 0.000000000\end{array}$

Al $\quad 2.257291000 \quad-1.303247000 \quad 0.513573000$

Al $\quad-0.000000000 \quad 0.000000000 \quad 3.969760000$

Al $\quad-0.000000000 \quad-2.606495000 \quad-0.513573000$

Al $\quad \begin{array}{llll}1.564359000 & -0.903183000 & -2.087897000\end{array}$

Al $\quad-0.000000000 \quad-1.806366000 \quad 2.087897000$

Al $\quad-1.564359000 \quad-0.903183000 \quad-2.087897000$

Al $\quad 0.000000000 \quad 0.000000000 \quad 0.000000000$

Al $\quad \begin{array}{llll}1.564359000 & 0.903183000 & 2.087897000\end{array}$

Al $\quad-0.000000000 \quad-0.000000000 \quad-3.969760000$

\section{- $\mathrm{Al}_{n} \mathrm{X}(n=11-15, \mathrm{X}=\mathrm{F}, \mathrm{Cl}, \mathrm{Br}$, and I $)$}

\begin{tabular}{lrrr}
\multicolumn{4}{l}{$\mathbf{A l}_{11} \mathbf{F}$} \\
$\mathrm{Al}$ & -1.311843000 & -1.461826000 & 1.301795000 \\
$\mathrm{Al}$ & 0.217712000 & 2.306634000 & 2.134467000 \\
$\mathrm{Al}$ & -1.311843000 & -1.461826000 & -1.301795000 \\
$\mathrm{Al}$ & -0.972086000 & 0.901361000 & -0.000000000 \\
$\mathrm{Al}$ & -1.216115000 & 0.411394000 & 3.098245000 \\
$\mathrm{Al}$ & -1.216115000 & 0.411394000 & -3.098245000 \\
$\mathrm{Al}$ & 0.217712000 & 2.306634000 & -2.134467000 \\
$\mathrm{Al}$ & 1.567841000 & 2.124017000 & -0.000000000 \\
$\mathrm{Al}$ & 1.120951000 & -0.201040000 & -1.409996000 \\
$\mathrm{Al}$ & 1.120951000 & -0.201040000 & 1.409996000 \\
$\mathrm{Al}$ & 0.773204000 & -2.404339000 & 0.000000000 \\
$\mathrm{~F}$ & 1.458354000 & -3.945300000 & 0.000000000
\end{tabular}

\begin{tabular}{|c|c|c|c|}
\hline \multicolumn{4}{|c|}{$\mathbf{A l}_{12} \mathbf{F}$} \\
\hline A1 & 1.829957000 & 0.458548000 & 2.192202000 \\
\hline $\mathrm{Al}$ & 3.174538000 & 0.369778000 & -0.000000000 \\
\hline 11 & -0.250638000 & 1.955977000 & -2.597180000 \\
\hline 18 & -2.065147000 & 0.599317000 & -1.306337000 \\
\hline $\mathrm{Al}$ & 0.343486000 & 1.226896000 & -0.000000000 \\
\hline$\pi$ & -0.250638000 & 1.955977000 & 2.597180000 \\
\hline $\mathrm{Al}$ & 1.18 & -1.4075 & -0.000000000 \\
\hline A & 1.829957000 & 0.458548000 & -2.192202000 \\
\hline $\mathrm{Al}$ & -0.250638000 & -1.112425000 & -2.215460000 \\
\hline A & -1.519798000 & -1.786519000 & 0.000000000 \\
\hline$\Delta$ & -2.065147000 & 0.599317000 & 1.306337000 \\
\hline $\mathrm{Al}$ & -0.250638000 & -1.112425000 & 2.215460000 \\
\hline & -2.475304000 & -3.185649000 & 0.000000000 \\
\hline
\end{tabular}




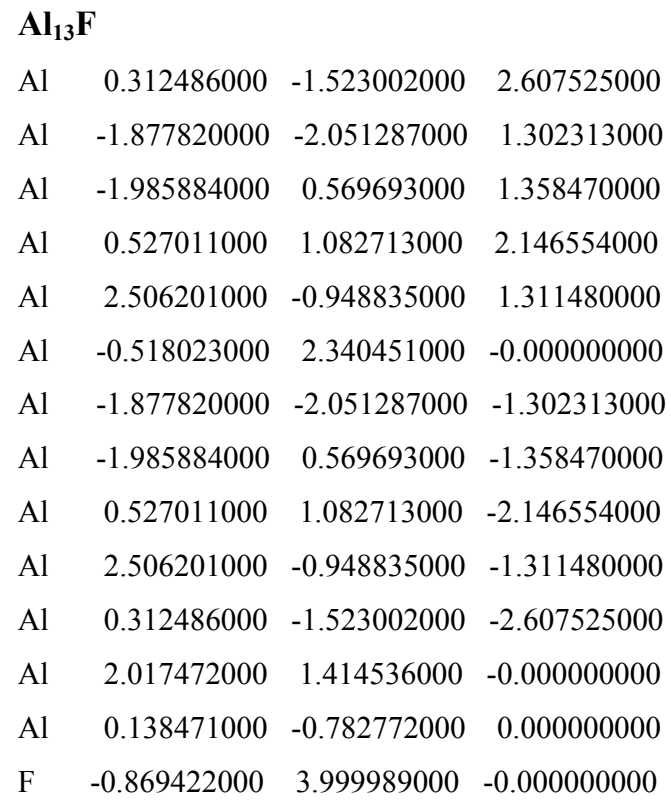

\section{$\mathrm{Al}_{14} \mathbf{F}$}

Al $\quad 0.043876000 \quad-3.204713000 \quad-0.000000000$

Al $\quad 2.230119000 \quad 0.597266000 \quad 1.503580000$

Al $\quad-0.003122000 \quad-1.114973000 \quad 2.103870000$

Al $\quad-0.001359000 \quad 0.431073000 \quad 0.000000000$

Al $\quad-0.003122000 \quad-1.114973000 \quad-2.103870000$

Al $\quad 2.230119000 \quad 0.597266000 \quad-1.503580000$

Al $\quad 1.366688000 \quad 2.726655000 \quad 0.000000000$

Al $\quad-0.003122000 \quad 1.758968000 \quad 2.375546000$

Al $\quad-2.239402000 \quad 0.618412000 \quad 1.479578000$

Al $\quad-1.915478000 \quad-1.541655000 \quad 0.000000000$

Al $\quad-0.003122000 \quad 1.758968000 \quad-2.375546000$

Al $\quad-1.397577000 \quad 2.807764000 \quad 0.000000000$

Al $\quad-2.239402000 \quad 0.618412000 \quad-1.479578000$

Al $\quad 1.960532000 \quad-1.552391000 \quad-0.000000000$

F $\quad-0.037018000 \quad-4.891003000 \quad-0.000000000$
Al $\quad-1.227234000 \quad-1.566498000 \quad-1.617709000$

Al $\quad \begin{array}{llll}1.225558000 & 2.048483000 & -1.662926000\end{array}$

Al $\quad-0.009651000 \quad 0.195628000 \quad-0.000000000$

Al $\quad-1.227234000 \quad-1.566498000 \quad 1.617709000$

Al $\quad 2.379609000 \quad 3.722374000 \quad-0.000000000$

F $\quad-1.698876000 \quad-5.183381000 \quad 0.000000000$

\begin{abstract}
$\mathrm{Al}_{12} \mathrm{Cl}$
Al $\quad 1.965369000 \quad 0.642594000 \quad 2.189154000$

Al $\quad 3.313231000 \quad 0.562348000 \quad 0.000000000$

Al $\quad-0.115289000 \quad 2.143208000 \quad-2.594720000$

Al $\quad-1.925262000 \quad 0.781047000 \quad-1.304050000$

Al $\quad 0.485827000 \quad 1.429772000 \quad-0.000000000$

Al $\quad-0.115289000 \quad 2.143208000 \quad 2.594720000$

Al $\quad \begin{array}{llll}1.320449000 & -1.210896000 & 0.000000000\end{array}$

Al $\quad \begin{array}{llll}1.965369000 & 0.642594000 & -2.189154000\end{array}$

Al $\quad-0.115289000 \quad-0.931417000 \quad-2.219373000$

Al $\quad-1.378346000 \quad-1.609548000 \quad-0.000000000$

Al $\quad-1.925262000 \quad 0.781047000 \quad 1.304050000$

Al $\quad-0.115289000 \quad-0.931417000 \quad 2.219373000$

$\mathrm{Cl} \quad-2.569580000 \quad-3.397235000 \quad-0.000000000$
\end{abstract}




$\begin{array}{lrrr}\mathrm{Al} & -2.182820000 & -1.926964000 & -1.301365000 \\ \mathrm{Al} & -1.857364000 & 0.679787000 & -1.361502000 \\ \mathrm{Al} & 0.709555000 & 0.765932000 & -2.149517000 \\ \mathrm{Al} & 2.320928000 & -1.572919000 & -1.311075000 \\ \mathrm{Al} & 0.062236000 & -1.771905000 & -2.606930000 \\ \mathrm{Al} & 2.238673000 & 0.841658000 & -0.000000000 \\ \mathrm{Al} & 0.016307000 & -1.014900000 & -0.000000000 \\ \mathrm{Cl} & -0.191255000 & 4.322539000 & 0.000000000\end{array}$

\begin{tabular}{lrrr}
\multicolumn{4}{l}{$\mathbf{A l}_{14} \mathbf{C l}$} \\
$\mathrm{Al}$ & -2.236687000 & 1.490384000 & -0.841206000 \\
$\mathrm{Al}$ & -0.000000000 & 2.107984000 & 0.871368000 \\
$\mathrm{Al}$ & 0.000000000 & 2.369501000 & -2.003595000 \\
$\mathrm{Al}$ & 0.000000000 & 0.000000000 & -0.674167000 \\
$\mathrm{Al}$ & -2.236687000 & -1.490384000 & -0.841206000 \\
$\mathrm{Al}$ & -1.922031000 & 0.000000000 & 1.312784000 \\
$\mathrm{Al}$ & 1.922031000 & -0.000000000 & 1.312784000 \\
$\mathrm{Al}$ & 2.236687000 & 1.490384000 & -0.841206000 \\
$\mathrm{Al}$ & 1.386889000 & -0.000000000 & -3.002856000 \\
$\mathrm{Al}$ & -1.386889000 & 0.000000000 & -3.002856000 \\
$\mathrm{Al}$ & -0.000000000 & -2.107984000 & 0.871368000 \\
$\mathrm{Al}$ & 2.236687000 & -1.490384000 & -0.841206000 \\
$\mathrm{Al}$ & -0.000000000 & -2.369501000 & -2.003595000 \\
$\mathrm{Al}$ & -0.000000000 & -0.000000000 & 2.984707000 \\
$\mathrm{Cl}$ & -0.000000000 & -0.000000000 & 5.122674000
\end{tabular}

\section{$\mathrm{Al}_{15} \mathrm{Cl}$}

$\begin{array}{lrrr}\mathrm{Al} & -1.162875000 & 1.254753000 & 2.277482000 \\ \mathrm{Al} & 1.277032000 & -0.313623000 & 2.249582000 \\ \mathrm{Al} & -2.617473000 & 0.642764000 & 0.000000000 \\ \mathrm{Al} & -0.996521000 & 2.878338000 & 0.000000000 \\ \mathrm{Al} & 1.246415000 & 2.299109000 & 1.669671000 \\ \mathrm{Al} & -1.162875000 & 1.254753000 & -2.277482000 \\ \mathrm{Al} & 1.330942000 & -1.865291000 & -0.000000000 \\ \mathrm{Al} & -1.308867000 & -3.320084000 & -0.000000000 \\ \mathrm{Al} & 1.277032000 & -0.313623000 & -2.249582000 \\ \mathrm{Al} & 2.699166000 & 0.594085000 & 0.000000000 \\ \mathrm{Al} & -1.162875000 & -1.348673000 & -1.600484000 \\ \mathrm{Al} & 1.246415000 & 2.299109000 & -1.669671000 \\ \mathrm{Al} & 0.039626000 & 0.437089000 & 0.000000000 \\ \mathrm{Al} & -1.162875000 & -1.348673000 & 1.600484000 \\ \mathrm{Al} & 2.396953000 & 3.971209000 & 0.000000000 \\ \mathrm{Cl} & -1.482931000 & -5.445657000 & -0.000000000\end{array}$

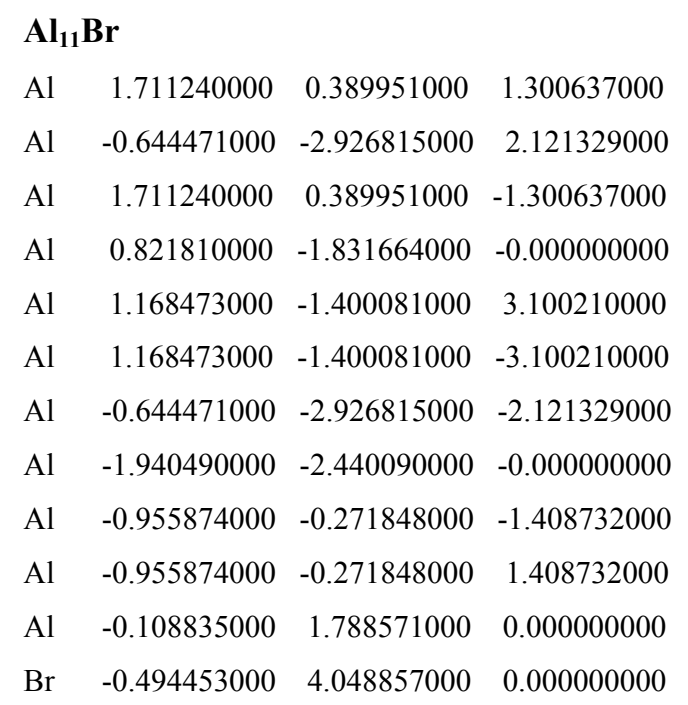

\section{$\mathbf{A l}_{12} \mathbf{B r}$}

Al $\quad-0.942349000 \quad-2.245458000 \quad 2.192605000$

Al $\quad-1.967664000 \quad-3.111075000 \quad-0.000000000$

Al $\quad 1.602125000 \quad-1.909648000 \quad-2.593442000$

Al $\quad \begin{array}{llll}1.987454000 & 0.324395000 & -1.304323000\end{array}$

Al $\quad 0.671896000 \quad-1.794109000 \quad-0.000000000$

Al $\quad 1.602125000 \quad-1.909648000 \quad 2.593442000$

Al $\quad-1.748773000 \quad-0.454753000 \quad-0.000000000$

Al $\quad-0.942349000 \quad-2.245458000 \quad-2.192605000$

Al $\quad-0.506987000 \quad 0.327001000 \quad-2.216195000$

Al $\quad-0.052617000 \quad 1.688984000 \quad 0.000000000$

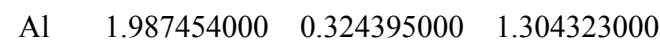

$\begin{array}{lllll}\text { Al } & -0.506987000 & 0.327001000 & 2.216195000\end{array}$

$\begin{array}{lllll}\mathrm{Br} & -0.439522000 & 3.966253000 & 0.000000000\end{array}$ 


\begin{abstract}
$\mathrm{Al}_{15} \mathbf{B r}$
Al $\quad-1.046716000 \quad 1.707630000 \quad 2.276844000$

Al $\quad 1.393316000 \quad 0.134683000 \quad 2.249950000$

Al $\quad-2.500171000 \quad 1.103756000 \quad 0.000000000$

Al $\quad-0.872612000 \quad 3.336925000 \quad 0.000000000$

Al $\quad 1.366252000 \quad 2.747942000 \quad 1.674525000$

Al $\quad-1.046716000 \quad 1.707630000 \quad-2.276844000$

Al $\quad 1.438509000 \quad-1.414816000 \quad-0.000000000$

Al $\quad-1.203165000 \quad-2.870247000 \quad-0.000000000$

Al $\quad 1.393316000 \quad 0.134683000 \quad-2.249950000$

Al $\quad 2.816784000 \quad 1.041313000 \quad 0.000000000$

Al $\quad-1.046716000 \quad-0.896175000 \quad-1.596580000$

Al $\quad 1.366252000 \quad 2.747942000 \quad-1.674525000$

Al $\quad 0.156980000 \quad 0.893702000 \quad 0.000000000$

Al $\quad-1.046716000 \quad-0.896175000 \quad 1.596580000$

Al $\quad 2.521953000 \quad 4.408981000 \quad 0.000000000$

$\mathrm{Br} \quad-1.370775000 \quad-5.158316000 \quad-0.000000000$
\end{abstract}

\section{$\mathbf{A l}_{11} \mathbf{I}$}

Al $\quad-0.707137000 \quad-3.341133000 \quad 2.113728000$

Al $\quad \begin{array}{llll}1.763468000 & -0.099909000 & 1.300258000\end{array}$

Al $\quad 1.763468000 \quad-0.099909000 \quad-1.300258000$

Al $\quad-0.707137000 \quad-3.341133000 \quad-2.113728000$

Al $\quad-1.997265000 \quad-2.801595000 \quad-0.000000000$
Al $\quad-0.931278000 \quad-0.672204000 \quad 1.412330000$

Al $\quad-0.019982000 \quad 1.354895000 \quad 0.000000000$

Al $\quad 1.148861000-1.869132000 \quad-3.097623000$

Al $\quad-0.931278000 \quad-0.672204000 \quad-1.412330000$

Al $\quad 1.148861000 \quad-1.869132000 \quad 3.097623000$

Al $\quad 0.791069000 \quad-2.287332000 \quad-0.000000000$

I $\quad-0.324178000 \quad 3.850646000 \quad 0.000000000$
$\mathbf{A l}_{13} \mathbf{I}$
Al $\quad 0.008879000 \quad-2.564266000 \quad 2.607195000$
Al $\quad-2.239960000 \quad-2.644056000 \quad 1.301001000$
Al $\quad-1.819922000 \quad-0.052576000 \quad 1.359441000$
Al $\quad 0.744711000 \quad-0.052605000 \quad 2.146729000$
Al $\quad 2.274439000 \quad-2.446109000 \quad 1.311545000$
Al $\quad-0.026567000 \quad 1.385049000 \quad-0.000000000$
Al $\quad-2.239960000 \quad-2.644056000 \quad-1.301001000$
Al $\quad-1.819922000 \quad-0.052576000 \quad-1.359441000$
Al $\quad 0.744711000 \quad-0.052605000 \quad-2.146729000$
Al $\quad 2.274439000 \quad-2.446109000 \quad-1.311545000$
Al $\quad 0.008879000 \quad-2.564266000 \quad-2.607195000$
Al $\quad 2.274066000 \quad-0.029340000 \quad 0.000000000$
Al $\quad-0.006715000 \quad-1.821737000 \quad 0.000000000$
I $\quad-0.043434000 \quad 3.920911000 \quad-0.000000000$
$\mathbf{A l}_{14} \mathbf{I}$
Al $\quad 0.000000000 \quad 0.000000000 \quad 2.116769000$
Al $\quad 2.238324000 \quad 1.486778000 \quad-1.717886000$
Al $\quad 0.000000000 \quad 2.098385000 \quad 0.005269000$ 


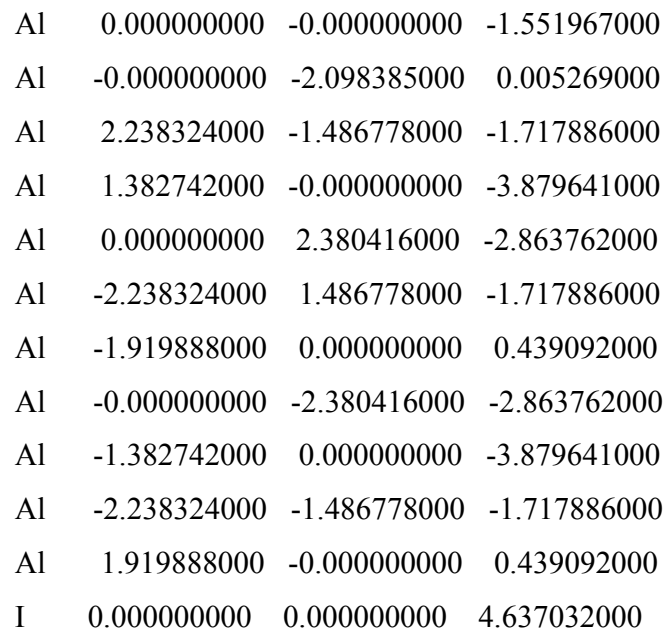

\begin{abstract}
$\mathbf{A l}_{15} \mathbf{I}$
Al $\quad-0.951013000 \quad 2.131114000 \quad 2.276721000$

Al $\quad 1.488006000 \quad 0.556611000 \quad 2.249945000$

Al $\quad-2.402475000 \quad 1.534353000 \quad 0.000000000$

Al $\quad-0.771633000 \quad 3.764675000 \quad-0.000000000$

Al $\quad 1.462928000 \quad 3.169810000 \quad 1.676126000$

Al $\quad-0.951013000 \quad 2.131114000 \quad-2.276721000$

Al $\quad 1.532298000 \quad-0.991943000 \quad 0.000000000$

Al $\quad-1.108344000 \quad-2.452484000 \quad 0.000000000$

Al $\quad 1.488006000 \quad 0.556611000 \quad-2.249945000$

Al $\quad 2.913195000 \quad 1.463210000 \quad-0.000000000$

Al $\quad-0.951013000 \quad-0.472517000 \quad-1.591371000$

Al $\quad \begin{array}{llll}1.462928000 & 3.169810000 & -1.676126000\end{array}$

Al $\quad 0.254453000 \quad 1.320700000 \quad-0.000000000$

Al $\quad-0.951013000 \quad-0.472517000 \quad 1.591371000$

Al $\quad 2.619709000 \quad 4.828425000 \quad-0.000000000$

I $\quad-1.259532000 \quad-4.963786000 \quad 0.000000000$
\end{abstract}

- $\mathrm{Al}_{n} \mathrm{X}_{2}(n=11-15, \mathrm{X}=\mathrm{F}, \mathrm{Cl}, \mathrm{Br}$, and I)

$$
\begin{array}{lrrr}
\text { Al } & -1.159400000 & 0.622994000 & 0.996757000 \\
\text { Al } & 1.190957000 & -1.728593000 & -0.553693000 \\
\text { Al } & 0.468832000 & 0.703701000 & -1.241460000 \\
\text { F } & 2.095088000 & -2.985211000 & -1.223990000 \\
\text { F } & 4.423658000 & 0.787414000 & -0.181051000
\end{array}
$$
$\mathrm{Al}_{13} \mathrm{~F}_{2}$
Al $\quad 2.713511000 \quad 0.138866000 \quad 0.000000000$
Al $\quad 1.188557000 \quad-0.744312000 \quad 2.314896000$
Al $\quad 1.188557000 \quad 1.869222000 \quad 1.505909000$
Al $\quad 1.188557000 \quad 1.869222000 \quad-1.505909000$
$\begin{array}{llll}\text { Al } & 1.188557000 & -0.744312000 & -2.314896000\end{array}$
Al $\quad-1.287152000 \quad 2.253616000 \quad-0.000000000$
$\begin{array}{llll}\text { Al } & -1.188557000 & 0.744312000 & 2.314896000\end{array}$
$\begin{array}{lllll}\mathrm{Al} & 0.000000000 & 0.000000000 & 0.000000000\end{array}$
Al $\quad-2.713511000 \quad-0.138866000 \quad 0.000000000$
Al $\quad-1.188557000 \quad-1.869222000 \quad-1.505909000$
Al $\quad-1.188557000 \quad-1.869222000 \quad 1.505909000$
$\begin{array}{lllll}\mathrm{Al} & -1.188557000 & 0.744312000 & -2.314896000\end{array}$
Al $\quad \begin{array}{llll}1.287152000 & -2.253616000 & -0.000000000\end{array}$
F $\quad-1.921195000 \quad 3.815924000 \quad-0.000000000$
F $\quad 1.921195000 \quad-3.815924000 \quad-0.000000000$ 


$\begin{array}{lrrr}\mathrm{Al} & 0.066229000 & 0.205165000 & 0.000000000 \\ \mathrm{Al} & 1.173523000 & 1.024240000 & 2.372326000 \\ \mathrm{Al} & -1.103320000 & 2.072151000 & 1.540480000 \\ \mathrm{Al} & -1.103320000 & 2.072151000 & -1.540480000 \\ \mathrm{Al} & -1.103320000 & -0.737847000 & -2.108913000 \\ \mathrm{Al} & 1.674834000 & -1.408338000 & -1.477437000 \\ \mathrm{Al} & 1.674834000 & -1.408338000 & 1.477437000 \\ \mathrm{Al} & 1.084659000 & 2.633766000 & 0.000000000 \\ \mathrm{Al} & 1.173523000 & 1.024240000 & -2.372326000 \\ \mathrm{Al} & 2.836704000 & 0.610770000 & 0.000000000 \\ \mathrm{Al} & -2.688404000 & 0.610687000 & 0.000000000 \\ \mathrm{~F} & 4.510215000 & 0.853988000 & 0.000000000 \\ \mathrm{~F} & -3.951436000 & -3.100315000 & 0.000000000\end{array}$
Al $\quad-0.123376000 \quad-0.718181000 \quad-1.240621000$
$\mathrm{Cl} \quad-2.301344000 \quad 3.128834000 \quad-0.873573000$
Cl $\quad-4.419852000 \quad-1.311146000 \quad-0.026003000$
$\mathrm{Al}_{13} \mathrm{Cl}_{2}$
Al $\quad 2.711798000 \quad 0.135182000 \quad 0.000000000$
Al $\quad \begin{array}{llll}1.188360000 & -0.745277000 & 2.311142000\end{array}$
Al $\quad 1.188360000 \quad 1.869491000 \quad 1.501984000$
Al $\quad \begin{array}{llll}1.188360000 & 1.869491000 & -1.501984000\end{array}$
Al $\quad 1.188360000 \quad-0.745277000 \quad-2.311142000$
Al $\quad-1.287532000 \quad 2.263613000 \quad-0.000000000$
Al $\quad-1.188360000 \quad 0.745277000 \quad 2.311142000$
Al $\quad 0.000000000 \quad 0.000000000 \quad 0.000000000$
Al $\quad-2.711798000 \quad-0.135182000 \quad 0.000000000$
Al $\quad-1.188360000 \quad-1.869491000 \quad-1.501984000$
Al $\quad-1.188360000 \quad-1.869491000 \quad 1.501984000$
Al $\quad-1.188360000 \quad 0.745277000 \quad-2.311142000$
Al $\quad 1.287532000 \quad-2.263613000 \quad-0.000000000$
$\mathrm{Cl} \quad-2.091195000 \quad 4.239318000 \quad-0.000000000$
$\begin{array}{llll}\mathrm{Cl} & 2.091195000 & -4.239318000 & -0.000000000\end{array}$ 


$\begin{array}{lrrr}\mathrm{Al} & -1.136761000 & 2.179587000 & 1.531533000 \\ \mathrm{Al} & -1.136761000 & 2.179587000 & -1.531533000 \\ \mathrm{Al} & -1.136761000 & -0.630984000 & -2.101391000 \\ \mathrm{Al} & 1.643828000 & -1.301115000 & -1.479201000 \\ \mathrm{Al} & 1.643828000 & -1.301115000 & 1.479201000 \\ \mathrm{Al} & 1.057897000 & 2.744840000 & 0.000000000 \\ \mathrm{Al} & 1.137020000 & 1.132300000 & -2.377985000 \\ \mathrm{Al} & 2.805338000 & 0.714293000 & 0.000000000 \\ \mathrm{Al} & -2.720069000 & 0.701544000 & 0.000000000 \\ \mathrm{Cl} & 4.929821000 & 1.022103000 & 0.000000000 \\ \mathrm{Cl} & -4.288482000 & -3.344842000 & 0.000000000\end{array}$

$\begin{array}{lrrr}\mathrm{Al}_{\mathbf{1 5}} \mathbf{C l}_{\mathbf{2}} & & & \\ \mathrm{Al} & -2.654349000 & 0.274744000 & 0.000000000 \\ \mathrm{Al} & -1.378186000 & 1.291756000 & 2.279905000 \\ \mathrm{Al} & -0.903614000 & -1.273616000 & -1.498367000 \\ \mathrm{Al} & -1.378186000 & 1.291756000 & -2.279905000 \\ \mathrm{Al} & -1.770895000 & 2.876092000 & 0.000000000 \\ \mathrm{Al} & 1.277538000 & 0.248637000 & -2.273426000 \\ \mathrm{Al} & 1.277538000 & 0.248637000 & 2.273426000 \\ \mathrm{Al} & -0.480461000 & -3.415105000 & 0.000000000 \\ \mathrm{Al} & 2.608243000 & 1.339141000 & 0.000000000 \\ \mathrm{Al} & 0.800655000 & 2.798718000 & 1.500389000 \\ \mathrm{Al} & 1.719415000 & -1.187835000 & 0.000000000 \\ \mathrm{Al} & 0.800655000 & 2.798718000 & -1.500389000 \\ \mathrm{Al} & -0.070011000 & 0.758314000 & 0.000000000 \\ \mathrm{Al} & -0.903614000 & -1.273616000 & 1.498367000 \\ \mathrm{Al} & -0.018439000 & 4.953263000 & 0.000000000 \\ \mathrm{Cl} & -1.100971000 & -5.454469000 & 0.000000000 \\ \mathrm{Cl} & 1.922045000 & -3.515228000 & 0.000000000\end{array}$

$\mathrm{Br} \quad-3.924851000 \quad-1.610566000 \quad 0.014261000$

$\begin{array}{lrrr}\mathrm{Al}_{13} \mathrm{Br}_{2} & & \\ \mathrm{Al} & 2.709451000 & 0.133967000 & 0.000000000 \\ \mathrm{Al} & 1.188132000 & -0.745332000 & 2.309804000 \\ \mathrm{Al} & 1.188132000 & 1.871585000 & 1.500465000 \\ \mathrm{Al} & 1.188132000 & 1.871585000 & -1.500465000 \\ \mathrm{Al} & 1.188132000 & -0.745332000 & -2.309804000 \\ \mathrm{Al} & -1.283095000 & 2.268719000 & -0.000000000 \\ \mathrm{Al} & -1.188132000 & 0.745332000 & 2.309804000 \\ \mathrm{Al} & 0.000000000 & 0.000000000 & 0.000000000 \\ \mathrm{Al} & -2.709451000 & -0.133967000 & 0.000000000 \\ \mathrm{Al} & -1.188132000 & -1.871585000 & -1.500465000 \\ \mathrm{Al} & -1.188132000 & -1.871585000 & 1.500465000 \\ \mathrm{Al} & -1.188132000 & 0.745332000 & -2.309804000 \\ \mathrm{Al} & 1.283095000 & -2.268719000 & -0.000000000 \\ \mathrm{Br} & -2.141860000 & 4.394406000 & -0.000000000 \\ \mathrm{Br} & 2.141860000 & -4.394406000 & -0.000000000\end{array}$

\section{$\mathrm{Al}_{13} \mathrm{Br}_{2}$}

$\mathrm{Al}_{12} \mathrm{Br}_{2}$

Al $\quad-2.117032000 \quad 0.623362000 \quad 2.252869000$

Al $\quad-2.972836000 \quad 1.482000000 \quad 0.000000000$

Al $\quad-2.117032000 \quad-1.939212000 \quad-2.626364000$

Al $\quad-0.017415000 \quad-2.671928000 \quad-1.300813000$

Al $\quad-1.723970000 \quad-0.944173000 \quad 0.000000000$

Al $\quad-2.117032000 \quad-1.939212000 \quad 2.626364000$

Al $\quad-0.068586000 \quad 1.380146000 \quad 0.000000000$

Al $\quad-2.117032000 \quad 0.623362000 \quad-2.252869000$

Al $\quad 0.333216000 \quad-0.169582000 \quad-2.155249000$

Al $\quad \begin{array}{llll}1.647848000 & -0.821448000 & 0.000000000\end{array}$

Al $\quad-0.017415000 \quad-2.671928000 \quad 1.300813000$

Al $\quad 0.333216000 \quad-0.169582000 \quad 2.155249000$

$\begin{array}{lllll}\mathrm{Br} & 0.123935000 & 3.693885000 & 0.000000000\end{array}$

$\mathrm{Br} \quad 3.944720000 \quad-1.012840000 \quad 0.000000000$ $\begin{array}{lll}\text { Br }-2.141860000 & -4.394406000 & -0.000000000\end{array}$

\footnotetext{
$\mathrm{Al}_{11} \mathrm{Br}_{2}$

Al $\quad 2.124081000 \quad 1.275061000 \quad-0.989206000$

Al $\quad-1.689130000 \quad-1.152172000 \quad 0.184601000$

Al $\quad 0.401413000 \quad-2.817220000 \quad 0.552180000$

Al $\quad 3.995821000 \quad-0.658164000 \quad-1.204576000$

Al $\quad 4.244079000 \quad 1.113540000 \quad 0.736660000$

Al $\quad \begin{array}{llll}1.753415000 & 1.708931000 & 1.653056000\end{array}$

Al $\quad 0.048994000 \quad-0.350423000 \quad 1.944433000$

Al $\quad 2.409647000 \quad-2.799488000 \quad-1.183184000$

Al $\quad 2.312034000 \quad-0.925984000 \quad 0.853514000$

Al $\quad-0.421414000 \quad 1.390651000 \quad-0.033768000$

Al $\quad 0.493025000 \quad-0.764378000 \quad-1.230819000$

$\mathrm{Br} \quad-1.896164000 \quad 3.088720000 \quad-0.490763000$
} 


$\begin{array}{lrrr}\mathrm{Al} & -1.188243000 & -0.452040000 & -2.100872000 \\ \mathrm{Al} & 1.589053000 & -1.123018000 & -1.476431000 \\ \mathrm{Al} & 1.589053000 & -1.123018000 & 1.476431000 \\ \mathrm{Al} & 1.002327000 & 2.931224000 & 0.000000000 \\ \mathrm{Al} & 1.084997000 & 1.309885000 & -2.377356000 \\ \mathrm{Al} & 2.750819000 & 0.897922000 & 0.000000000 \\ \mathrm{Al} & -2.771048000 & 0.887821000 & 0.000000000 \\ \mathrm{Br} & 5.037454000 & 1.222442000 & 0.000000000 \\ \mathrm{Br} & -4.444113000 & -3.293863000 & 0.000000000\end{array}$

\section{$\mathbf{A l}_{15} \mathbf{B r}_{2}$}

$\begin{array}{lrrr}\mathrm{Al} & -1.609265000 & 2.824902000 & 0.000000000 \\ \mathrm{Al} & -0.804076000 & 1.308096000 & 2.274033000 \\ \mathrm{Al} & -0.804076000 & 1.308096000 & -2.274033000 \\ \mathrm{Al} & 0.618862000 & 3.476821000 & -1.506420000 \\ \mathrm{Al} & 0.618862000 & 3.476821000 & 1.506420000 \\ \mathrm{Al} & 2.048375000 & 1.244417000 & -2.279727000 \\ \mathrm{Al} & 0.618862000 & -0.936038000 & 1.492342000 \\ \mathrm{Al} & -0.539173000 & -2.795171000 & 0.000000000 \\ \mathrm{Al} & 2.836750000 & -0.181111000 & 0.000000000 \\ \mathrm{Al} & 2.048375000 & 1.244417000 & 2.279727000 \\ \mathrm{Al} & 0.618862000 & -0.936038000 & -1.492342000 \\ \mathrm{Al} & 3.026067000 & 2.558379000 & 0.000000000 \\ \mathrm{Al} & 0.637638000 & 1.265265000 & 0.000000000 \\ \mathrm{Al} & -1.765822000 & 0.149781000 & 0.000000000 \\ \mathrm{Al} & 2.203927000 & 5.140264000 & 0.000000000 \\ \mathrm{Br} & -0.628426000 & -5.089845000 & 0.000000000 \\ \mathrm{Br} & -2.994550000 & -2.022604000 & 0.000000000\end{array}$

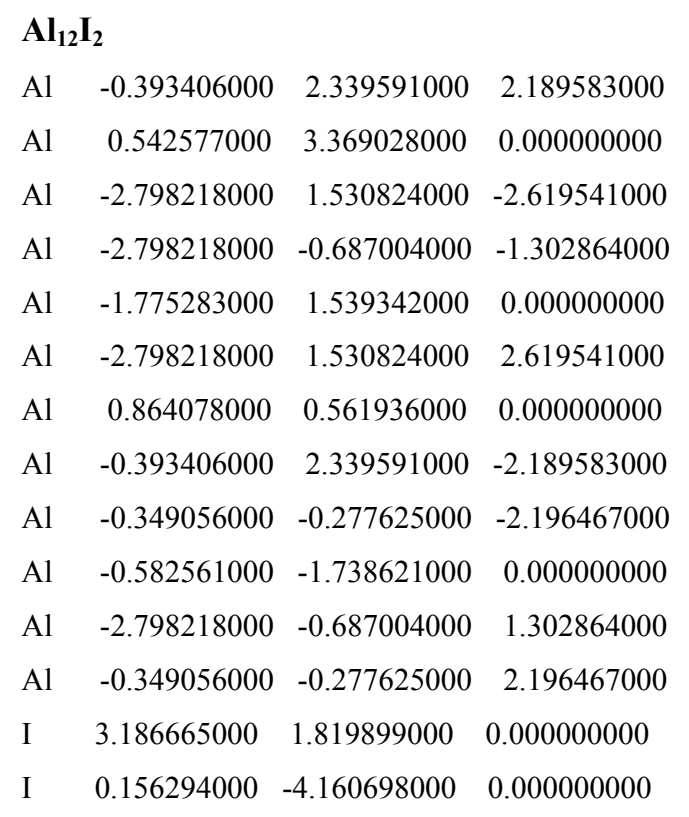

\footnotetext{
$\mathbf{A l}_{11} \mathbf{I}_{2}$

Al $\quad 2.620046000 \quad 1.128432000 \quad-0.923315000$

Al $\quad-1.214261000 \quad-1.269884000 \quad 0.191195000$

Al $\quad 0.857853000 \quad-2.984986000 \quad 0.453259000$

Al $\quad 4.477708000 \quad-0.808503000 \quad-1.243674000$

Al $\quad 4.758304000 \quad 0.874154000 \quad 0.767584000$

Al $\quad 2.291758000 \quad 1.470085000 \quad 1.740038000$

Al $\quad 0.569488000 \quad-0.583076000 \quad 1.959496000$

Al $\quad 2.846889000 \quad-2.916182000 \quad-1.307970000$

Al $\quad 2.807177000 \quad-1.143217000 \quad 0.814875000$

Al $\quad 0.084476000 \quad 1.245495000 \quad 0.063770000$

Al $\quad 0.954924000 \quad-0.866799000 \quad-1.241746000$

I $\quad-1.489009000 \quad 3.167933000 \quad-0.335316000$

I $\quad-3.675268000 \quad-1.731928000 \quad 0.022946000$
}

\begin{abstract}
$\mathbf{A l}_{13} \mathbf{I}_{2}$
Al $\quad 2.707746000 \quad 0.131843000 \quad 0.000000000$

Al $\quad \begin{array}{llll}1.188282000 & -0.744380000 & 2.308216000\end{array}$

Al $\quad 1.188282000 \quad 1.875170000 \quad 1.496612000$

Al $\quad 1.188282000 \quad 1.875170000 \quad-1.496612000$

Al $\quad 1.188282000 \quad-0.744380000 \quad-2.308216000$

Al $\quad-1.276531000 \quad 2.273100000 \quad-0.000000000$

Al $\quad-1.188282000 \quad 0.744380000 \quad 2.308216000$

Al $\quad 0.000000000 \quad 0.000000000 \quad 0.000000000$

Al $\quad-2.707746000 \quad-0.131843000 \quad 0.000000000$

Al $\quad-1.188282000 \quad-1.875170000 \quad-1.496612000$

Al $\quad-1.188282000 \quad-1.875170000 \quad 1.496612000$

Al $\quad-1.188282000 \quad 0.744380000 \quad-2.308216000$

Al $\quad \begin{array}{llll}1.276531000 & -2.273100000 & -0.000000000\end{array}$

I $\quad-2.214104000 \quad 4.605704000 \quad-0.000000000$

I $\quad 2.214104000 \quad-4.605704000 \quad-0.000000000$
\end{abstract}




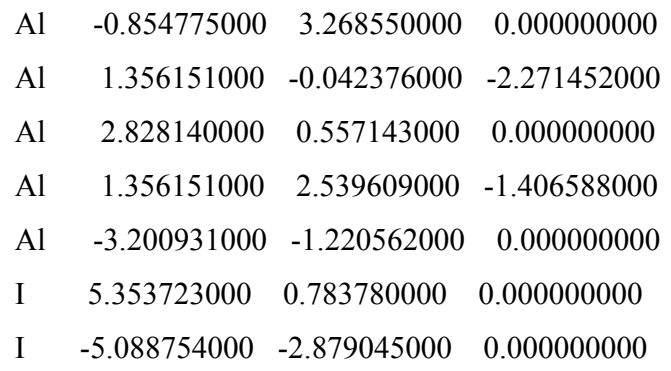

\section{$\mathbf{A l}_{15} \mathbf{I}_{2}$}

$\begin{array}{lrrr}\text { Al } & 3.300480000 & 1.498609000 & 0.000000000 \\ \text { Al } & 1.802260000 & 0.660584000 & 2.274417000 \\ \text { Al } & 1.802260000 & 0.660584000 & -2.274417000 \\ \text { Al } & 4.005337000 & -0.709521000 & -1.512138000 \\ \text { Al } & 4.005337000 & -0.709521000 & 1.512138000 \\ \text { Al } & 1.802260000 & -2.193190000 & -2.278927000 \\ \text { Al } & -0.406910000 & -0.808480000 & 1.485428000 \\ \text { Al } & -2.357193000 & 0.205100000 & 0.000000000 \\ \text { Al } & 0.399163000 & -3.013908000 & 0.000000000 \\ \text { Al } & 1.802260000 & -2.193190000 & 2.278927000 \\ \text { Al } & -0.406910000 & -0.808480000 & -1.485428000 \\ \text { Al } & 3.139520000 & -3.145833000 & 0.000000000 \\ \text { Al } & 1.800673000 & -0.783857000 & 0.000000000 \\ \text { Al } & 0.621377000 & 1.598242000 & 0.000000000 \\ \text { Al } & 5.686501000 & -2.256890000 & 0.000000000 \\ \text { I } & -4.874376000 & 0.014845000 & 0.000000000 \\ \text { I } & -1.747386000 & 2.928491000 & 0.000000000\end{array}$

- $\mathrm{Al}_{n} \mathrm{I}^{-}, \mathrm{Al}_{n} \mathrm{I}_{2}^{-}$, and $\mathrm{Al}_{n} \mathrm{I}_{3}{ }^{-}(n=11-15)$

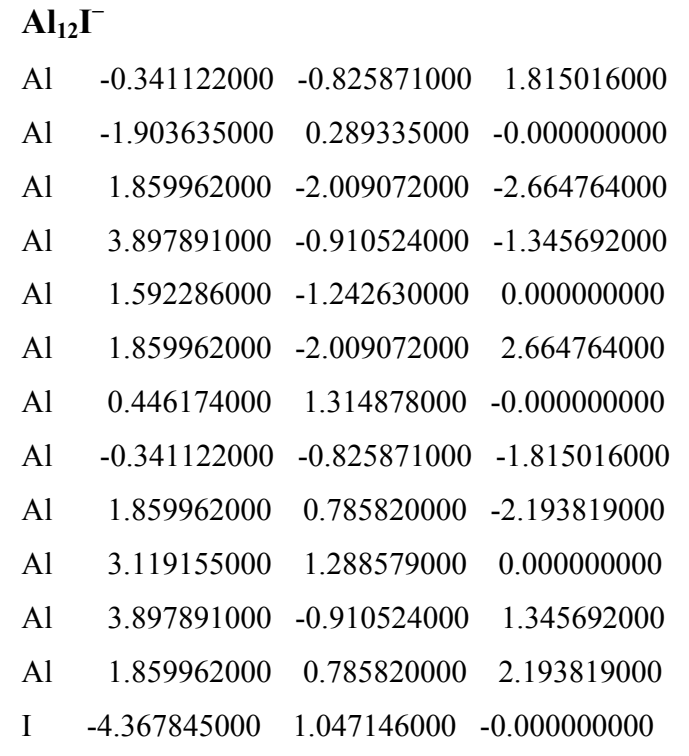

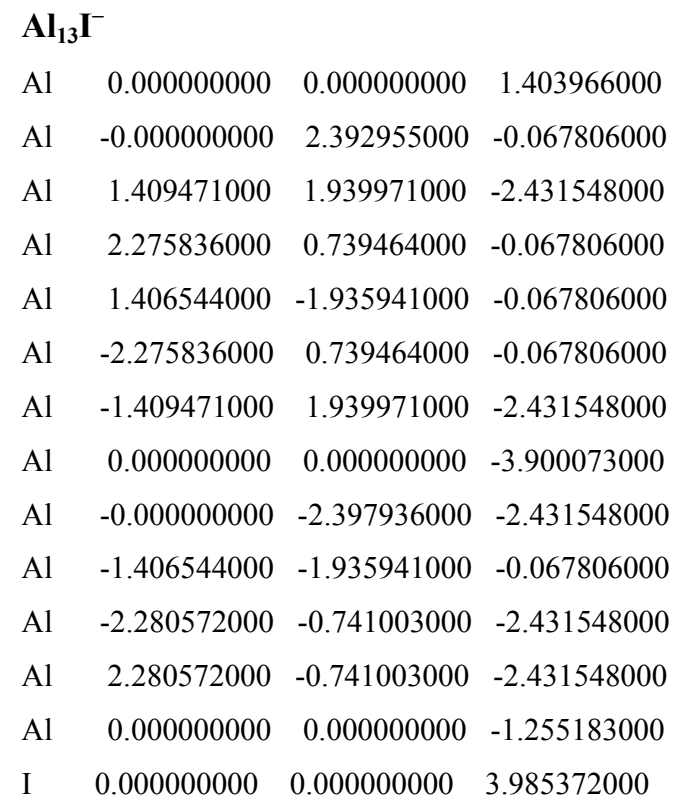

\section{$\mathbf{A l}_{14} \mathbf{I}^{-}$}

Al $\quad 2.660470000 \quad 0.856161000 \quad 1.420346000$

Al $\quad-0.890764000 \quad-1.451839000 \quad 0.000000000$

$\begin{array}{llll}\mathrm{Al} & 1.838008000 & -1.379283000 & 0.000000000\end{array}$

$\begin{array}{lllll}\text { Al } & 0.444933000 & 0.937962000 & 0.000000000\end{array}$

Al $\quad 0.418497000 \quad 2.290923000 \quad 2.280520000$

$\begin{array}{lllll}\text { Al } & -1.817226000 & 0.910984000 & 1.368438000\end{array}$

Al $\quad-1.817226000 \quad 0.910984000 \quad-1.368438000$

$\begin{array}{llll}\text { Al } & 0.418497000 & -0.516335000 & -2.253102000\end{array}$

$\begin{array}{lllll}\text { Al } & 2.660470000 & 0.856161000 & -1.420346000\end{array}$

$\begin{array}{llll}\text { Al } & 4.481704000 & 2.582759000 & 0.000000000\end{array}$ 


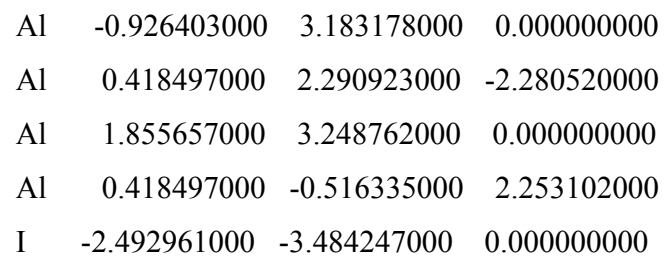

\begin{abstract}
$\mathbf{A l}_{15} \mathbf{I}^{-}$
Al $\quad-2.397723000 \quad 1.345410000 \quad 0.000000000$

Al $\quad-0.941443000 \quad 2.102114000 \quad 2.261840000$

Al $\quad-0.941443000 \quad-0.525742000 \quad-1.565596000$

Al $\quad-0.941443000 \quad 2.102114000 \quad-2.261840000$

$\begin{array}{llll}\text { Al } & -0.987859000 & 3.727401000 & 0.000000000\end{array}$

Al $\quad \begin{array}{llll}1.469449000 & 0.653949000 & -2.253363000\end{array}$

Al $\quad 1.469449000 \quad 0.653949000 \quad 2.253363000$

Al $\quad-0.658729000 \quad-2.556482000 \quad-0.000000000$

Al $\quad 2.918519000 \quad 1.460141000 \quad 0.000000000$

Al $\quad 1.447562000 \quad 3.283942000 \quad 1.551748000$

Al $\quad \begin{array}{llll}1.572661000 & -0.976418000 & -0.000000000\end{array}$

Al $\quad 1.447562000 \quad 3.283942000 \quad-1.551748000$

Al $\quad 0.248440000 \quad 1.328885000 \quad 0.000000000$

Al $\quad-0.941443000 \quad-0.525742000 \quad 1.565596000$

Al $\quad 1.293785000 \quad 5.460666000 \quad 0.000000000$

I $\quad-0.995197000 \quad-5.106333000 \quad-0.000000000$
\end{abstract}

Al $\quad-1.695976000 \quad 2.598242000 \quad-0.467857000$

Al $\quad 2.331535000 \quad 1.317065000 \quad-1.722947000$

Al $\quad 2.089889000 \quad-0.565461000 \quad 0.061256000$

Al $\quad-0.231215000 \quad-0.422112000 \quad 1.381252000$

Al $\quad-2.375300000 \quad 0.055543000 \quad-0.015061000$

Al $\quad 0.406022000 \quad 3.095582000 \quad-1.973365000$

Al $\quad-0.168424000 \quad 0.369660000 \quad-1.296546000$

Al $\quad-1.544185000 \quad 1.828037000 \quad 2.133660000$

Al $\quad 0.672086000 \quad 2.227364000 \quad 0.672187000$

I $\quad 2.908517000 \quad-2.994511000 \quad-0.246792000$

I $\quad-4.640128000 \quad-1.138036000 \quad-0.186023000$

\begin{tabular}{lllllllll}
\multicolumn{2}{|c}{$\mathbf{A l}_{11} \mathbf{I}_{2}^{-}$} & & Al & 0.000000000 & 0.000000000 & 0.000000000 \\
$\mathrm{Al}$ & 1.481417000 & 2.112591000 & 2.092283000 & I & 0.000000000 & 0.000000000 & -5.179517000 \\
$\mathrm{Al}$ & 1.481417000 & -2.112591000 & 2.092283000 & I & -0.000000000 & -0.000000000 & 5.179517000
\end{tabular}

Al $\quad-1.481417000 \quad-2.112591000 \quad 2.092283000$

Al $\quad-1.481417000 \quad 2.112591000 \quad 2.092283000$

Al $\quad 0.000000000 \quad 2.256591000 \quad-0.018362000$

Al $\quad 1.448801000 \quad-0.000000000 \quad 0.300663000$

Al $\quad-0.000000000 \quad-2.256591000 \quad-0.018362000$

Al $\quad-2.986709000 \quad 0.000000000 \quad 2.687639000$

Al $\quad-1.448801000 \quad 0.000000000 \quad 0.300663000$

Al $\quad 2.986709000 \quad-0.000000000 \quad 2.687639000$

Al $\quad 0.000000000 \quad 0.000000000 \quad 2.714762000$

I $\quad 0.000000000 \quad-3.766646000 \quad-2.087821000$

I $\quad 0.000000000 \quad 3.766646000 \quad-2.087821000$

\section{$\mathrm{Al}_{14} \mathbf{I}_{2}^{-}$}

$\begin{array}{lrrr}\mathrm{Al}_{\mathbf{1 3}} \mathbf{I}_{\mathbf{2}}^{-} & & & \\ \mathrm{Al} & 0.000000000 & 2.399733000 & 1.174944000 \\ \mathrm{Al} & -1.410527000 & 1.941425000 & -1.174944000 \\ \mathrm{Al} & 1.410527000 & 1.941425000 & -1.174944000 \\ \mathrm{Al} & 2.282281000 & 0.741558000 & 1.174944000 \\ \mathrm{Al} & 0.000000000 & 0.000000000 & 2.623742000 \\ \mathrm{Al} & 2.282281000 & -0.741558000 & -1.174944000 \\ \mathrm{Al} & -2.282281000 & 0.741558000 & 1.174944000 \\ \mathrm{Al} & 0.000000000 & 0.000000000 & -2.623742000 \\ \mathrm{Al} & -0.000000000 & -2.399733000 & -1.174944000 \\ \mathrm{Al} & -1.410527000 & -1.941425000 & 1.174944000 \\ \mathrm{Al} & -2.282281000 & -0.741558000 & -1.174944000 \\ \mathrm{Al} & 1.410527000 & -1.941425000 & 1.174944000 \\ \mathrm{Al} & 0.000000000 & 0.000000000 & 0.000000000 \\ \mathrm{I} & 0.000000000 & 0.000000000 & -5.179517000 \\ \mathrm{I} & -0.000000000 & -0.000000000 & 5.179517000\end{array}$

$\begin{array}{lrrr}\text { Al } & -1.278491000 & -1.593967000 & 0.000000000 \\ \text { Al } & 1.018877000 & -1.114474000 & 1.564348000 \\ \text { Al } & -1.319918000 & 0.042359000 & 2.264125000 \\ \text { Al } & -0.027895000 & 0.774905000 & 0.000000000 \\ \text { Al } & -1.319918000 & 0.042359000 & -2.264125000 \\ \text { Al } & 1.018877000 & -1.114474000 & -1.564348000 \\ \text { Al } & 2.653699000 & 1.097090000 & 0.000000000 \\ \text { Al } & 1.058661000 & 1.549663000 & 2.267614000 \\ \text { Al } & -1.319918000 & 2.671854000 & 1.474028000 \\ \text { Al } & -2.790281000 & 0.698813000 & 0.000000000 \\ \text { Al } & 1.058661000 & 1.549663000 & -2.267614000 \\ \text { Al } & 0.937945000 & 3.253694000 & 0.000000000 \\ \text { Al } & -1.319918000 & 2.671854000 & -1.474028000 \\ \text { Al } & 3.090211000 & -1.461714000 & 0.000000000\end{array}$


I $\quad 5.003870000 \quad-3.161217000 \quad 0.000000000$

I $\quad-5.362129000 \quad 0.937083000 \quad 0.000000000$

\footnotetext{
$\mathrm{Al}_{11} \mathbf{I}^{-}$

Al $\quad 2.195891000 \quad-2.747454000 \quad 0.083947000$

Al $\quad-1.185910000 \quad 0.356148000 \quad 1.130717000$

Al $\quad 0.880027000 \quad 1.456794000 \quad 2.332505000$

Al $\quad 3.678225000 \quad-1.873761000 \quad 2.105386000$

Al $\quad 4.659041000 \quad-1.432165000 \quad-0.364398000$

Al $\quad 2.348044000 \quad-0.691562000 \quad-1.597708000$

Al $\quad 0.705598000 \quad 1.260177000 \quad-0.490815000$

Al $\quad 2.514784000 \quad-0.093329000 \quad 3.675908000$

Al $\quad 2.837403000 \quad 0.341292000 \quad 0.840350000$

Al $\quad-0.103707000 \quad-1.294024000 \quad-0.715277000$

Al $\quad 0.840256000 \quad-1.284645000 \quad 1.771550000$

I $\quad-1.832105000 \quad-2.698779000 \quad-1.985282000$

I $\quad-3.659176000 \quad 0.728238000 \quad 1.692820000$

I $\quad 0.740234000 \quad 3.442860000 \quad-1.859201000$
}

$$
\begin{array}{lrrr}
\text { Al } & -0.756989000 & 0.121793000 & 2.158869000 \\
\text { Al } & 3.311128000 & -0.270621000 & 0.106580000 \\
\text { Al } & 1.842931000 & -0.441142000 & -2.037424000 \\
\text { Al } & -0.681575000 & -0.052264000 & -1.857263000 \\
\text { Al } & -1.617548000 & 1.582597000 & 0.003502000 \\
\text { Al } & 1.828974000 & -0.027683000 & 2.309959000 \\
\text { Al } & 0.913856000 & 1.075206000 & 0.042018000 \\
\text { Al } & -2.108601000 & -1.098467000 & 0.193085000 \\
\text { Al } & 0.403499000 & -1.598179000 & 0.358386000 \\
\text { I } & -4.347330000 & -2.327763000 & 0.225990000 \\
\text { I } & 5.595408000 & 0.932392000 & -0.021673000 \\
\text { I } & -3.284675000 & 3.518669000 & -0.166629000
\end{array}
$$
$\mathrm{Al}_{14} \mathbf{I}_{3}^{-}$
Al $\quad 1.106887000 \quad 2.425478000 \quad 1.627745000$
Al $\quad-1.563371000 \quad 2.002383000 \quad-0.000000000$
Al $\quad-0.909868000 \quad 0.538985000 \quad 2.258927000$
Al $\quad 0.492932000 \quad 0.406420000 \quad-0.000000000$
Al $\quad 2.999080000 \quad 1.147848000 \quad-0.000000000$
Al $\quad \begin{array}{llll}1.106887000 & 2.425478000 & -1.627745000\end{array}$
Al $\quad-0.909868000 \quad 0.538985000 \quad-2.258927000$
Al $\quad-2.017943000 \quad-0.661663000 \quad 0.000000000$
Al $\quad-0.059595000 \quad-1.931972000 \quad 1.451487000$
Al $\quad 1.833089000 \quad-0.113041000 \quad 2.277278000$
Al $\quad 1.833089000 \quad-0.113041000 \quad-2.277278000$
Al $\quad-0.059595000 \quad-1.931972000 \quad-1.451487000$ 

Al $\quad 2.336914000 \quad-1.507983000 \quad 0.000000000$
Al $\quad 0.089284000 \quad 4.144761000 \quad-0.000000000$
I $\quad-0.437397000 \quad-4.201678000 \quad-2.610623000$
I $\quad-0.665073000 \quad 6.595456000 \quad-0.000000000$
I $\quad-0.437397000 \quad-4.201678000 \quad 2.610623000$
$\mathrm{Al}_{15} \mathbf{I}_{3}^{-}$
Al $\quad-1.530081000 \quad 0.472344000 \quad 0.000000000$
Al $\quad-0.035924000 \quad 1.113875000 \quad 2.229359000$
Al $\quad-0.034660000 \quad-1.518630000 \quad-1.551868000$
Al $\quad-0.035924000 \quad 1.113875000 \quad-2.229359000$
Al $\quad-0.042713000 \quad 2.737624000 \quad 0.000000000$
Al $\quad 2.351092000 \quad-0.436627000 \quad-2.286119000$
Al $\quad 2.351092000 \quad-0.436627000 \quad 2.286119000$
Al $\quad 0.426026000 \quad-3.575423000 \quad 0.000000000$
Al $\quad 3.969921000 \quad 0.316232000 \quad 0.000000000$
Al $2.351092000 \quad 2.132653000 \quad 1.462324000$
Al $\quad 2.553354000 \quad-2.073846000 \quad 0.000000000$
Al $\quad 2.351092000 \quad 2.132653000 \quad-1.462324000$
Al $\quad 1.213866000 \quad 0.197020000 \quad 0.000000000$
Al $\quad-0.034660000 \quad-1.518630000 \quad 1.551868000$
Al $\quad 4.554631000 \quad 3.013431000 \quad 0.000000000$
I $\quad-0.131693000 \quad-6.069106000 \quad 0.000000000$
I $\quad-4.051861000 \quad-0.026849000 \quad 0.000000000$
I $\quad-0.822232000 \quad 5.195785000 \quad 0.000000000$ 